32

33

34

35

36

37

\title{
AIM2 Inflammasome Mediates Hallmark Neuropathological Alterations and Cognitive Impairment in a Mouse Model of Vascular Dementia
}

\author{
Luting Poh ${ }^{1,2}$, David Y. Fann ${ }^{1,3,{ }^{\star}}$, Peiyan Wong ${ }^{2,4}$, Hong Meng Lim ${ }^{1}$, Sok Lin Foo ${ }^{1}$,
} Sung-Wook Kang ${ }^{1}$, Vismitha Rajeev ${ }^{1,2}$, Sharmelee Selvaraji ${ }^{1,2}$, Vinaya Rajagopal lyer ${ }^{2}$, Nageiswari Parathy ${ }^{2}$, Mohammad Badruzzaman Khan ${ }^{5}$, David C. Hess ${ }^{5}$, Dong-Gyu $\mathrm{Jo}^{6}$, Grant R. Drummond ${ }^{7}$, Christopher G. Sobey ${ }^{7}$, Mitchell K.P. Lai ${ }^{2,8}$, Christopher LiHsian Chen ${ }^{2,8}$, Lina H. K. Lim¹, Thiruma V. Arumugam,6,7,*

${ }^{1}$ Department of Physiology, Yong Loo Lin School of Medicine, National University of Singapore, Singapore

${ }^{2}$ Department of Pharmacology, Yong Loo Lin School of Medicine, National University of Singapore, Singapore

${ }^{3}$ Department of Biochemistry, Yong Loo Lin School of Medicine, National University of Singapore, Singapore

${ }^{4}$ Neuroscience and Behavioural Disorders Programme, Duke-NUS Medical School, Singapore

${ }^{5}$ Department of Neurology, Medical College of Georgia, Augusta University, Augusta, GA, USA

${ }^{6}$ School of Pharmacy, Sungkyunkwan University, Suwon, Republic of Korea

${ }^{7}$ Department of Physiology, Anatomy and Microbiology, La Trobe University, Bundoora, VIC, Australia

${ }^{8}$ Memory, Aging and Cognition Centre, National University Health System, Singapore

${ }^{*}$ Correspondence: Thiruma V. Arumugam, Department of Physiology, Anatomy and Microbiology, La Trobe University, Bundoora, VIC, Australia. E-mail: g.arumugam@latrobe.edu.au; David Y. Fann, Department of Biochemistry, Yong Loo Lin School of Medicine, National University of Singapore, Singapore. E-mail: bchfywd@nus.edu.sg 


\section{Abstract}

42 Chronic cerebral hypoperfusion is associated with vascular dementia (VaD). 43 Cerebral hypoperfusion may initiate complex molecular and cellular inflammatory 44 pathways that contribute to long-term cognitive impairment and memory loss. Here 45 we used a bilateral common carotid artery stenosis (BCAS) mouse model of $\mathrm{VaD}$ to 46 investigate its effect on the innate immune response - particularly the inflammasome 47 signaling pathway. Comprehensive analyses revealed that chronic cerebral 48 hypoperfusion induces a complex temporal expression and activation of inflammasome components and their downstream products (IL-1 $\beta$ and IL-18) in 50 different brain regions, and promotes activation of apoptotic and pyroptotic cell death 51 pathways. Polarized glial cell activation, white matter lesion formation and 52 hippocampal neuronal loss also occurred in a spatiotemporal manner. Moreover, in 53 AIM2 knockout mice we observed attenuated inflammasome-mediated production of 54 proinflammatory cytokines, apoptosis and pyroptosis, as well as resistance to chronic microglial activation, myelin breakdown, hippocampal neuronal loss, and behavioural and cognitive deficits following BCAS. Hence, we have demonstrated that activation of the AIM2 inflammasome substantially contributes to the pathophysiology of chronic cerebral hypoperfusion-induced brain injury and may therefore represent a promising therapeutic target for attenuating cognitive impairment in $\mathrm{VaD}$.

\section{Introduction}

Vascular dementia $(\mathrm{VaD})$ is the second most common form of dementia that is associated with vascular diseases resulting in dysfunction and damage to the cerebral vasculature, which causes disruption of cerebral blood flow, brain injury, and ultimately cognitive impairment and memory loss ${ }^{1}$. Clinical findings in $\mathrm{VaD}$ include chronic cerebral hypoperfusion, white matter lesions (WMLs), strokes, hippocampal atrophy, decline in executive functioning and memory impairment ${ }^{2}$. Recent evidence indicates that chronic cerebral hypoperfusion is associated with systemic inflammation before WMLs and neurological symptoms develop in $\mathrm{VaD}^{3-5}$. Interleukin

71 1-beta (IL-1 $\beta$ ), a potent cytokine that orchestrates inflammatory pathways, is likely to 72 be a key contributor to $\mathrm{VaD}^{5}$. The primary molecular machinery for IL-1 $\beta$ production 73 in response involves assembly and activation of the inflammasome. 
74

75

76

77

78

79

80

81

82

83

84

85

86

87

88

89

90

91

92

93

94

95

96

97

98

100

101

102

103

104

105

106

107

Inflammasome is an intracellular multi-protein complex that initiates an innate immune response, and is involved in multiple acute and chronic neurological diseases such as ischemic stroke, Alzheimer's disease (AD), Parkinson's Disease (PD), and amyotrophic lateral sclerosis $(A L S)^{6-11}$. In the canonical inflammasome pathway, stimulation and homo-oligomerization of inflammasome receptors such as nucleotide-binding domain and leucine-rich repeat containing (NLR) family pyrin domain containing (NLRP) 1 (NLRP1), NLRP3, NLR family CARD domain containing 4 (NLRC4) and absent in melanoma 2 (AIM2) initiates formation of the inflammasome complex, recruitment and activation of adaptor (ASC) and effector (total caspase-1 and -8) inflammasome components into biologically active cleaved caspases- 1 and $-8^{6,12}$. In the noncanonical inflammasome pathway, caspase-11 is activated via auto-proteolysis upon dimerization to produce cleaved caspase-11. Upon inflammasome assembly and activation, activity of cleaved caspase- 1 and -8 are increased and downstream proinflammatory cytokines such as mature IL-1 $\beta$ and IL-18 are generated ${ }^{6}$. This leads to activation of glial cells, endothelial dysfunction, and oligodendrocyte injury and demyelination, eventually resulting in neurovascular unit disintegration, neuronal loss and brain circuit dysfunction. Cleaved caspase-1 and -8 may also promote cell death by activating apoptosis and pyroptosis pathways $^{13-14}$. Cleaved caspase-1 and -8 induces apoptosis and pyroptosis by catalyzing the cleavage of total caspase-3 and full-length Gasdermin D (GSDMD-FL) into cleaved caspase-3 and N-terminal Gasdermin D (GSDMD-NT), respectively. GSDMD-NT can oligomerize with other $\mathrm{N}$-terminal units and cause pore formation in the nuclear and plasma membranes, leading to leakage of cellular content and proinflammatory cytokines ${ }^{15,16}$. GSDMD activation can also be facilitated directly by non-canonical cleaved caspase- $11^{17}$. The inhibition of caspase- 1 was demonstrated to prevent pyroptosis in experimental models of multiple sclerosis by reducing demyelination and neurodegeneration ${ }^{18}$. It was also established that the inflammasome complex also contributed to cognitive decline in a APP/PS1 mouse model of Alzheimer's disease ${ }^{9,19}$. Specifically, it was shown that deletion of the AIM2 inflammasome in that AD model promoted dendrite branching and synaptic plasticity, and improvement in spatial memory ${ }^{20}$. AIM2 is known to play a role in the host immune response to microbial $D N A^{21}$; however, several reports have established that endogenous double-stranded DNA (dsDNA) can also activate AIM2 to form the AIM2 
108 inflammasome ${ }^{22,23}$. Moreover, AIM2 also responds to dsDNA released from damaged 109 host cells and is a potential ligand that can activate AIM2 in neurons and glial cells 110 following chronic hypoperfusion $22,24$.

111

112 While evidence for direct involvement of the inflammasome complex in $\mathrm{VaD}$ is 113 lacking, a cytokine profile of plasma from $\mathrm{VaD}$ patients found $\mathrm{IL}-1 \beta$ to be the most 114 abundant ${ }^{25-28}$. It was also reported that IL-1 $\beta$ impeded oligodendrocyte recruitment 115 and inhibited white matter repair and functional recovery during the early stages of 116 chronic cerebral hypoperfusion ${ }^{5}$. However, any potential involvement of 117 inflammasome activation in chronic cerebral hypoperfusion-induced glial activation, 118 white matter damage, neuronal cell death and cognitive dysfunction has not been 119 studied.

120

121 In the present study, we have comprehensively investigated the role of the 122 inflammasome signaling pathway in the bilateral common carotid artery stenosis 123 (BCAS) mouse model of $\mathrm{VaD}$. The BCAS mouse model has been well established in 124 imitating a wide range of neurological clinical conditions of white matter rarefaction, 125 hippocampal atrophy and cognitive decline induced by chronic cerebral 126 hypoperfusion in humans ${ }^{29,30}$. Moreover, BCAS develops white matter lesions without 127 cerebral infarctions and optic nerve damage, while hippocampal neuronal death and 128 atrophy is induced making it the most appropriate animal model in studying $\mathrm{VaD}^{31}$. 129 Using the BCAS mouse model, we report an increased expression and activation of 130 inflammasome complexes, production of mature proinflammatory cytokines, 131 polarized glial activation, WMLs, and neuronal cell death occurring in a time132 dependent manner in the cerebral cortex, hippocampus and striatum following BCAS. 133 Notably, these brain regions exhibited distinct profiles of inflammasome expression 134 and activation, and a specific prominent increase of AIM2 in the cerebral cortex and 135 hippocampus. Furthermore, we demonstrated that genetic deletion of AIM2 protects 136 against BCAS-induced inflammasome activation, proinflammatory cytokine 137 production, polarized glial activation, WML formation and neuronal cell death, 138 ultimately leading to an improvement in cognitive outcome. Overall, our findings 139 reveal that the AIM2 inflammasome may be a potential therapeutic target in VaD. 


\section{Materials and Methods}

\section{Experimental Animals and Genotyping}

144 All in vivo experimental procedures were approved by the National University of

145 Singapore, Singapore Animal Care and Use Committee and performed according to 146 the guidelines set forth by the National Advisory Committee for Laboratory Animal 147 Research (NACLAR), Singapore. Mice were housed in individual cages under 148 standard laboratory conditions. All efforts were made to minimize suffering and 149 numbers of animals used. All sections of the manuscript were performed and 150 reported in accordance with ARRIVE (Animal Research: Reporting In Vivo 151 Experiments) guidelines. Fourteen to sixteen weeks old wild type (WT) male 152 C57BL/6 mice (weighing 24 to 30 grams) were obtained from In Vivos, Singapore. 153 The AIM2 knockout (B6.129P2-Aim2<Gt(CSG445)Byg>/J; AIM2 KO) mice were 154 generated by Ingenious Targeting Laboratory (Ronkonkoma, NY) on the C57BL/6 155 background mice via targeted replacement of the AIM2 coding region with a 156 neomycin resistance gene through homologous recombination that was kindly 157 obtained from our collaborator Professor Jenny P. Y. Ting ${ }^{32}$. Primer sequences used 158 for PCR-based AIM2 deficient mouse genotyping are as follows: neomycin (KO) 159 forward, GGAACTTCGCTAGACTAGTACGCGTG; neomycin (KO) reverse, 160 CAACATTGTACAGATTGAGCAGG; AIM2 (WT) forward, 161 GATGGAGAGTGAGTACCGGGAAATGCTGTT; and AIM2 (WT) reverse,

162 TCTGCAAGTAGATTGGAGACAGACTCTGGTGA, resulting in a 450-bp band for the 163 wild type and a 250-bp band for the targeted AIM2 knockout when PCR analysis was 164 performed on the genomic DNA (Supplementary Fig. 1). All mice were given free 165 access to food and water ad libitum. The experimental groups consisted of 20-25 166 animals in each group.

167

\section{Bilateral Common Carotid Artery Stenosis (BCAS) Mouse Model}

169 Animals were anaesthetized with isoflurane and subjected to BCAS, and 170 subsequent chronic hypoperfusion using microcoils specifically designed for the 171 mouse (microcoil specifications: piano wire diameter $0.08 \mathrm{~mm}$, internal diameter $1720.18 \mathrm{~mm}$, coiling pitch $0.5 \mathrm{~mm}$, and total length $2.5 \mathrm{~mm}$; Sawane Spring Co Ltd, Japan) 173 was described elsewhere ${ }^{33}$. The left and right common carotid arteries (CCAs) were 174 exposed individually, freed from their sheaths, and a microcoil was twined by rotating 
175 it around each CCA. The site of surgery was closed, and the mice were observed

176 and taken care of post-surgery until conscious and recovered to freely access food

177 and water ad libitum. To study the disease progression, the experimental animals

178 were divided into eight time-point groups: Sham, 1, 3, 7, 15, 21, 30 and 60-days

179 BCAS. Sham animals were given a skin incision and their CCAs were exposed. All

180 animals were euthanized at their respective end-point after BCAS for subsequent

181 analysis. For biochemical analysis involving Wild Type control and AIM2 KO mice,

182 the animals were divided into three experimental groups: Sham, 15 and 30-days

183 BCAS. However, only the Sham and 30-days timepoints were selected to challenge

184 the cognitive ability of both WT and AIM2 KO mice during behavioral analysis.

185

\section{Measurements of Cerebral Blood Flow by Laser Speckle Contrast Imager}

187 High-resolution Laser Speckle Contrast Imager (PSI system, Perimed Inc.) was 188 used to image cerebral blood perfusion and record cerebral blood flow (CBF) before 189 BCAS (baseline), immediately after BCAS surgery and finally at their respective end190 points of BCAS. As shown in Supplementary Fig. 2, regions of interest (ROIs) 191 between the bregma and lambda were selected for overall perfusion in the area of 192 two hemispheres. Body temperature was maintained at $37 \pm 0.5^{\circ} \mathrm{C}$, and the skull 193 was shaved and exposed by a midline skin incision. The skull was cleaned gently 194 with sterile phosphate buffered saline (PBS) using a cotton applicator. Finally, the 195 image area was kept moist and a non-toxic silicon oil was applied on the skull, which 196 improved imaging. Perfusion images were acquired using the PSI system with a $19770 \mathrm{~mW}$ built-in laser diode for illumination and a $1388 \times 1038$ pixels CCD camera 198 installed $10 \mathrm{~cm}$ above the skull (speed $19 \mathrm{~Hz}$, and exposure time $6 \mathrm{mSec}$ ). Acquired 199 images were analyzed for changes in CBF (cerebral perfusion) using a dedicated 200 PIMSoft program (Perimed Inc.).

201

\section{Sample Collection and Processing}

203 Following each time points, mice were euthanized by administering a lethal dose 204 of inhaled carbon dioxide $\left(\mathrm{CO}_{2}\right)$ and the brains harvested. The different brain areas 205 (cerebral cortex, hippocampus and striatum) were immediately separated on ice and 206 were frozen in dry ice for tissue biochemical analysis ( $n=7-8$ in each experimental 207 group). A separate group of animals were sacrificed for histological analysis. Mice 208 were deeply anaesthetized, and perfusion fixation though the heart was performed 
209 with $25 \mathrm{~mL}$ of chilled 1 XPBS ( $\mathrm{pH} 7.4$ ) then followed by $25 \mathrm{~mL}$ chilled paraformaldehyde 210 (4\%). Once the perfusion was completed, the brains were harvested and placed in 211 vials containing $4 \%$ paraformaldehyde solution for immersion-fixation overnight at $212 \quad 4^{\circ} \mathrm{C}(\mathrm{n}=5-7$ in each experimental group).

213

214 Immunoblot Analysis

215 Immunoblot analysis was performed as described by us previously ${ }^{34}$. Briefly, brain 216 tissues were homogenized in lysis buffer and then combined with $2 \times$ Laemelli buffer 217 (Bio-Rad Laboratories, Inc., Hercules, CA, USA). Protein samples were then 218 separated on 7.5 to $12.5 \% \mathrm{v} / \mathrm{v}$ sodium dodecyl sulfate (SDS) gels. The SDS-PAGE 219 gels were transferred onto nitrocellulose membranes to probe for proteins. Next, the 220 nitrocellulose membranes were incubated with the following primary antibodies: 221 NLRP1 (Santa Cruz, sc390133), NLRP3 (Adipogen, AG20B0014), NLRC4 (Millipore, 222 06-1125), AIM2 (Cell Signaling, \#13095), IL-1 (Genetex, GTX74034), IL-18 223 (Biovision, 5180R-100), Total Caspase-1 and Cleaved Caspase-1 (p33 subunit) 224 (Adipogen, AG20B0042), Cleaved Caspase-1 (p20 subunit) (Cell Signaling, \#67314), 225 Casapse-8 (Cell Signaling, \#4927), Caspase-11 (Cell Signaling, \#14340), Total 226 Caspase-3 (Cell Signaling, \#9662), Cleaved Caspase-3 (Cell Signaling, \#9664), 227 GSDMD (Cell Signaling, \#93709), GSDMD-NT (Cell Signaling, \#50928), GSDME 228 (Santa Cruz, sc393162), IBA-1 (Abcam, ab5076), GFAP (Cell Signaling, \#12389), 229 and $\beta$-actin (Sigma-Aldrich, A5441) overnight at $4^{\circ} \mathrm{C}$ with agitation. Following primary 230 antibody incubation, membranes were washed three times with 1xTBST before 231 incubating with horseradish peroxidase (HRP)-conjugated secondary antibodies 232 (Goat Anti-Rabbit - Cell Signaling Technology, Danvers, MA, USA; Goat Anti-Mouse 233 - Sigma-Aldrich, St. Louis, MO, USA; Goat Anti-Rat - GE Healthcare Life Sciences, 234 Little Chalfont, UK) for $1 \mathrm{hr}$ at $24^{\circ} \mathrm{C}$ with agitation. Following secondary antibody 235 incubation, membranes were washed three times with 1XTBST, each time for $10 \mathrm{~min}$. 236 The substrate for HRP, enhanced chemiluminescence (ECL) (Bio-Rad Laboratories, 237 Inc., Hercules, CA, USA) was applied before the membranes were imaged using 238 ChemiDocXRS+imaging system (Bio-Rad Laboratories, Inc., Hercules, CA, USA). 239 Quantification of proteins was conducted using Image J software (Version 1.46; 240 National Institute of Health, Bethesda, MD, USA), where protein densitometry was 241 expressed relative to the densitometry of the corresponding $\beta$-actin. 


\section{Luxol Fast Blue and Cresyl Violet Staining}

243 Mouse brain tissues were fixed in 10\% neutral buffered formalin and then 244 processed into paraffin wax blocks. Coronal sections (4 $\mu \mathrm{m}$ thick) were obtained via 245 microtome sectioning. Axonal fibre density of Luxol-fast-blue (LFB) staining was 246 performed to detect the severity of white matter (WM) lesions. Briefly, de-waxed 247 rehydrated sections were immersed in the LFB solution (Abcam, UK) at $37^{\circ} \mathrm{C}$ 248 overnight. Excess staining was removed by $95 \%$ ethanol treatment followed by 249 washing with deionized water. Grey and white matter differentiation was initiated with 250 the treatment of $0.05 \%$ aqueous lithium carbonate (Abcam, UK) for 20 seconds, 251 followed by $70 \%$ ethanol until the nuclei are decolorized. Sections were immersed in 252 Cresyl Violet solution (Abcam, UK) for $5 \mathrm{~min}$ and washed in deionized water. The 253 sections were dehydrated in an ethanol gradient $(70-100 \%)$, and finally cleared in 254 xylene and mounted with a mounting agent. The bright field images were taken under $2554 \mathrm{X}$ and 60X magnification using an Olympus upright Fluorescence Microscope BX53. 256 The WM lesions were evaluated in five brain regions: the optic tract, internal capsule, 257 caudoputamen, corpus callosum (Medial) and corpus callosum (Paramedian). The 258 severity of the WM lesions was graded as normal (grade 0), disarrangement of the 259 nerve fibres (grade 1), the formation of marked vacuoles (grade 2), and the 260 disappearance of myelinated fibres (grade 3). The severity of neuropathology was 261 scored by three blinded examiners and the number of neurons in CA1, CA2 and CA3 262 were counted as previously described ${ }^{35}$. The general morphology and the 263 neurodegeneration in the hippocampus of BCAS animals were assessed by Cresyl 264 violet staining.

265

266

267

\section{Immunofluorescence Analysis}

268 immunostained with primary antibodies against Cleaved Caspase-1 (Affinity

269 Biosciences, AF4022), Cleaved Caspase-3 (Cell Signaling, \#9661), GSDMD (Santa 270 Cruz, sc393581), MBP (Cell Signaling, \#78896), MAP2 (Millipore, MAB3418), GFAP 271 (Cell Signaling, \#3670; \#12389), IBA-1 (Wako, 016-26721; Cell Signaling, \#17198), 272 CD86 (Santa Cruz, sc-28347), CD206 (eBioscience, 12-2069-42), C3 (Santa Cruz, 273 sc-28294), S100S10 (Invitrogen, PA5-95505), OLIG2 (R\&D System, AF2418) and 274 PECAM-1 (BD Pharmingen, 553370). Images were captured with an Olympus 275 FluoView FV1000 (Olympus, Japan) laser scanning confocal microscope using 
$27620 x / 0.70$ air objective and 100x/1.45 oil objective, with 488nm Argon ion and 543nm

$277 \mathrm{HeNe}$ laser as the excitation source. Single confocal images were converted to $512 \mathrm{x}$

278512 pixel 12-bit TIFF images.

279

280 Behavioral Paradigm and Training of BCAS Animals

281 Visual Acuity Test: To assess visual detection, mice were placed on a platform 282 within a rotating drum covered with black and white vertical stripes at consistent 283 spatial frequency ${ }^{36,37}$. The automated drum was then rotated in a clockwise direction 284 for $30 \mathrm{sec}$, and subsequently an anti-clockwise direction for $30 \mathrm{sec}$, after a $15 \mathrm{sec}$ inter285 trial interval. Both larger stripes $\left(2^{\circ}\right)$ and smaller stripes $\left(1^{\circ}\right)$ were tested for all 286 animals before conducting other behavioral tests.

287 Open Field Test: Locomotor activity was measured using an open field test ${ }^{38}$. 288 Each subject was placed in the center of the open field apparatus $(20 \times 20 \times 40 \mathrm{~cm}$; 289 Clever Sys Inc.; VA, USA). Total distance traveled $(\mathrm{cm})$ and average speed $(\mathrm{mm} / \mathrm{s})$ 290 were recorded and tracked via TopScan (Clever Sys Inc.; VA, USA). Data was 291 collected for 35 minutes.

292 Morris Water Maze: Morris water maze was performed 3 weeks after surgery 293 (sham or BCAS), as described previously ${ }^{39}$. Mice were trained 4 times a day at 10294 min intervals for 5 consecutive days in this fixed platform training. In each trial, mice 295 were given 60 secs to find the platform. Activity of the animal in the water maze was 296 video-tracked by EthoVision software (Noldus Information Technology, Leesburg, 297 VA), and the escape latency was recorded and analyzed. Long term spatial memory 298 was tested on the $6^{\text {th }}$ day, whereby the platform was removed, and each animal was 299 given 60secs in the water maze after being released from four different directions. 300 Time spent around the platform, number of visits to the targeted quadrant and time 301 spent in the targeted quadrant on the probe day were tracked and analyzed. The probe day was designed to fall between 32 to 34 days of BCAS surgery.

303

\section{Double Stranded DNA Measurement}

305 Double stranded DNA from the serum of BCAS animals were measured as 306 previously described using Quant-iTTM PicoGreen ® dsDNA Reagent and Kits. Briefly, $30720 \mu \mathrm{L}$ of plasma sample collected was diluted with $1 \mathrm{X}$ TE solution. Subsequently, 308 equal volume of working solution of Quant-iTTM PicoGreen reagent was added. It was 309 then protected from light and incubated for 5 minutes at room temperature. Samples 
310 were excited at $485 \mathrm{~nm}$ and fluorescence intensity was measured at 520nm using a

311 Synergy HT multi-detection microplate reader (BioTek, Winnooski, VT).

312

\section{Statistical Analysis}

314 Experimental data were analyzed by GraphPad Prism 5.02 and 8.0 software 315 (GraphPad Software, San Diego, CA, USA). All values are expressed as mean \pm 316 standard error of the mean (S.E.M). One-way Analysis of Variance (ANOVA) was 317 used, followed by a Bonferroni post-hoc test to determine differences between 318 groups. A P-value $<0.05$ was deemed to be statistically significant. For behavioral 319 data, non-parametric Kruskal-Wallis test was used to determine differences between 320 groups. A P-value $<0.05$ was deemed to be statistically significant.

321

322

\section{Results}

\section{Chronic cerebral hypoperfusion increases inflammasome signalling}

We first evaluated the protein expression of inflammasome receptors over 60 days of BCAS in the cerebral cortex, hippocampus and striatum (Supplementary Figs. 3, 4

327 \& 5). NLRP3, AIM2 and NLRC4 were all increased in the cerebral cortex, while only 328 AIM2 receptor expression was increased in the hippocampus compared to sham 329 controls (Supplementary Fig. 3a-d, 4). NLRP1 expression was increased in the 330 striatum while other inflammasome receptors remained unchanged (Supplementary 331 Figs. $5 a$ \& b). Expression of total caspase-8 and precursor IL-1 $\beta$ were increased in 332 the cerebral cortex after BCAS, whereas a decrease in precursor IL-18 was evident, 333 and ASC and total caspases-1 and -11 were unchanged (Supplementary Fig. 3e \& f). 334 In the hippocampus, expression of total caspases-1, -8 -11 and precursor IL-18 335 increased, whereas ASC was decreased after BCAS (Supplementary Fig. $3 g$ \& h). 336 ASC, total caspase-8 and precursors for IL-1 $\beta$ and IL-18 were also increased in the 337 striatum following BCAS (Supplementary Fig. 5c \& d).

To assess canonical inflammasome activation, expression of cleaved caspase-1 340 subunits ( $\mathrm{p} 33$, transient active unit; p20, final by-product) and -8 were examined. 341 After BCAS, elevated expression of cleaved caspase-1 (p20) and -8 was detected in 342 the cerebral cortex (Fig. 1a \& b) and also hippocampus (Fig. 1c \& d). Mature IL-1 $\beta$ 
343 and IL-18 - direct downstream markers of inflammasome activation - were also

344 higher in the cerebral cortex and hippocampus after BCAS versus sham controls 345 (Fig. 1a-d). Expression of non-canonical cleaved caspase-11 was also elevated in 346 the cerebral cortex and hippocampus after BCAS (Fig. 1a-d), whereas only cleaved 347 caspase-8, IL-1 $\beta$ and IL-18 were increased in the striatum (Supplementary Fig. 6). 348 Overall, the data reveal that inflammasome activation occurs in the brain in a spatial349 temporal manner as a result of chronic cerebral hypoperfusion.

\section{Chronic cerebral hypoperfusion promotes apoptosis and pyroptosis}

We next examined cell death mechanisms associated with inflammasome activation. Thus, we assessed the expression of cleaved caspase-3 and GSDMD-NT, 354 along with their precursor proteins after BCAS (Fig. 1e-h; Supplementary Fig. 7 \& 8). 355 Our data showed that in the cerebral cortex (Fig. 1e \& f) and hippocampus (Fig. 1g \&

$356 \mathrm{~h}$ ) the expression of cleaved caspase-3 and GSDMD-NT were increased following 357 BCAS compared to sham controls. Pyroptotic cell death (i.e. GSDMD-NT expression) 358 was more prominent in the cerebral cortex following BCAS (Fig. 1e \& f), whereas 359 only apoptosis (i.e. increased cleaved caspase-3 expression) was detected in the 360 striatum following BCAS (Supplementary Fig. 8).

361

We also assessed inflammasome-associated secondary pyroptosis/necrosis, 363 which can be induced by cleaved caspase-3, as indicated by expression of $\mathrm{N}$ 364 Terminal Gasdermin E (GSDME-NT). Similar to GSDMD-NT, inflammasome365 generated GSDME-NT can permeabilize nuclear and plasma membranes and 366 mitochondria, linking inflammasome activation to cell death ${ }^{16}$. We found GSDME-NT 367 to be increased in the cerebral cortex, but not in the hippocampus or striatum 368 following BCAS (Supplementary Fig. 7 \& 8). Expression patterns of inflammasome369 related proteins and pathways following BCAS-induced chronic cerebral 370 hypoperfusion are summarized in Supplementary Fig. 9. Overall, the data indicate 371 that BCAS induces differential priming of inflammasome components, inflammasome 372 activation, and induction of apoptosis and pyroptosis pathways in the brain in a 373 spatial-temporal manner. 
376 Chronic cerebral hypoperfusion induces glial cell activation, white matter 377 lesions and hippocampal neuronal death

378 We next analyzed for associations between inflammasome activity and hallmark 379 VaD pathologies involving glial cell activation, white matter integrity and neuronal 380 loss. Expression of ionized calcium binding adaptor molecule 1 (Iba1) and glial 381 fibrillary acidic protein (GFAP) indicated activation of microglia and astrocytes, 382 respectively (Fig. 2a-d). Following BCAS, we found increased Iba-1 in the cerebral 383 cortex (Fig. 2a \& b) and increased GFAP in the hippocampus (Fig. 2c \& d), and both 384 markers increased in the striatum (Supplementary Fig. 10a \& b). Subsequently, 385 colocalization of both Iba1 and GFAP with respective polarization markers CD86, 386 CD206, complement component 3 (C3) and S100A10 revealed activation of mainly 387 M1 (CD86 positive) and M2 (CD206 positive) microglia upon chronic cerebral 388 hypoperfusion in the cerebral cortex and hippocampus (Fig. 2e-h; Supplementary 389 Fig. 11a-h) and striatum (Supplementary Fig. 10c-f). Myelin basic protein (MBP) and 390 Luxol fast blue staining revealed myelin integrity, while cresyl violet and microtubule391 associated protein 2 (MAP2) staining enabled assessment of neuronal loss in the 392 hippocampus. Staining for MBP and MAP2 was reduced in the cerebral cortex (Fig. 393 2i \& k), hippocampus (Fig. 2j \& I) and striatum (Supplementary Fig. $10 \mathrm{~g} \mathrm{\&} \mathrm{h)} \mathrm{following}$ 39430 days of BCAS.

395

396

White matter integrity of five brain regions was assessed after 7-60 days of 397 hypoperfusion, and found to be disrupted in the corpus callosum (Paramedian and 398 Medial), caudoputamen, internal capsule and optic tract (Fig. 2m). All five areas 399 exhibited time-dependent rarefaction of white matter (Fig. 2n) from 7 days, although 400 the white matter lesion (WML) index was not increased in the optic tract until 60 days 401 of BCAS (Fig. 2m \& n). BCAS-induced neuronal loss was evident in hippocampal 402 CA1, CA2 and CA3 areas (Fig. 20-r). Whereas hippocampal sections from sham 403 controls showed normal neuronal cell bodies with distinct nuclei, nucleoli, and 404 densely packed neurons in all three hippocampal areas, there were signs of 405 widespread neuronal loss evident after BCAS (Fig. 20-r). Degeneration was more 406 pronounced in the hippocampal CA2 and CA3 areas with severe atrophy from 7 days 407 (Fig. 2o-r).

408 
409 AlM2 activation mediates apoptosis and pyroptosis during chronic cerebral 410 hypoperfusion

411 As AIM2 was upregulated in the cerebral cortex and hippocampus, we postulated

412 its involvement in BCAS-induced brain injury. Analysis of serum revealed an increase

413 in cell-free double stranded DNA (dsDNA) levels following BCAS, especially after 15 414 days (Fig. 3a). As dsDNA is the only known ligand that activates the AIM2 receptor $^{22,}$ $415^{24}$, the finding supports a potential involvement of the AIM2 inflammasome in BCAS416 induced brain injury.

To further examine the role of AIM2 inflammasome activation on injury following chronic cerebral hypoperfusion, mice with AIM2 deficiency (AIM2 KO) were studied

420 (Supplementary Fig. 1). We first confirmed via laser speckle contrast imaging that cerebral blood flow was equivalent under basal conditions and reduced following BCAS surgery in wild-type (WT) and AIM2 KO mice (Supplementary Fig. 12). This was similar to previously published data from BCAS in mice ${ }^{29}$. This BCAS-induced reduction in flow was sustained to a similar degree for at least 15 days in both genotypes but was slightly higher in AIM2 KO than WT mice at 30 days 426 (Supplementary Fig. 12a-c).

We found no difference in the basal expression levels of cleaved caspase-1, -8, and -11 , and both mature IL-1 $\beta$ and IL-18 (Fig. 3b-e) or in the levels of total caspases

$430-1,-8$ and -11 , or IL-1 $\beta$ and IL-18 precursors in the cerebral cortex and hippocampus 431 of WT and AIM2 KO mice (Supplementary Fig. 13a-d). However, there was evidence 432 of reduced inflammasome activation following BCAS in AIM2 KO mice when 433 compared to WT control (Fig. 3b-e). Of the three active effector proteins, only 434 canonical cleaved caspase-1 expression was reduced in AIM2 KO mice compared to 435 WT control following BCAS (Fig. 3b-e; Supplementary Fig. 14a \& b). There was also 436 reduced levels of mature IL-1 $\beta$ and IL-18 in the cerebral cortex and hippocampus of 437 AIM2 KO mice at 15 and 30 days following BCAS (Fig. 3b-e; Supplementary Fig. 14a 438 \& b). These data thus indicated less inflammasome activity occurs after BCAS in the 439 absence of the AIM2 receptor, especially mediated by canonical cleaved caspase-1. 440 Furthermore, protein expression of both cleaved caspase-3 and GSDMD-NT was 441 lower at 30 days after BCAS in the cerebral cortex (Fig. $3 f$ \& g) and hippocampus 
442 (Fig. 3h \& i) of AIM2 KO mice, indicating AIM2 inflammasome mediating apoptosis 443 and pyroptosis under chronic cerebral hypoperfusion.

Immunofluorescence studies indicated that cleaved caspase-1 (CC1) activity was lower in AIM2 KO mice in cortical (Fig. 3j; Supplementary Fig. 15a-e \& 16a-e) and 447 hippocampal (Fig. 3k; Supplementary Fig. 15f-j \& 16f-j) neurons (MAP2 positive) and 448 microglia (lba-1 positive) compared to WT controls at 30 days following BCAS. 449 Immunoreactivity against cleaved caspase-1 was similar in cortical and hippocampal 450 oligodendrocytes (OLIG2 positive), astrocytes (GFAP positive) and endothelial cells 451 (PECAM-1 positive) of WT and AIM2 KO mice. Cellular specificity of AIM2 452 inflammasome-mediated cell death following BCAS was assessed by 453 immunoreactivity against cleaved caspase-3 (CC3) and GSDMD in the cerebral 454 cortex (Fig. 3j; Supplementary Fig. 15a-e; Supplementary Fig.17a-e \& 18a-e) and 455 hippocampus (Fig. 3k; Supplementary Fig. 15f-j; Supplementary Fig.17f-j \& 18f-j). 456 The data indicate that pro-apoptotic cleaved caspase-3 immunoreactivity was lower 457 in cortical (Fig. 3j; Supplementary Fig. 15a-e \& 17a-e) and hippocampal (Fig. 3k; 458 Supplementary Fig. 15f-j \& 17f-j) neurons (MAP2 positive) and microglia (Iba-1 459 positive) of AIM2 KO versus WT mice. Similarly, immunoreactivity of pro-pyroptotic 460 GSDMD was reduced in neurons and microglia in the cortex (Fig. 3j; Supplementary 461 Fig. 15a-e \& 18a-e) and hippocampus (Fig. 3k; Supplementary Fig. 15f-j \& 18f-j) of 462 AIM2 KO mice. These findings were consistent with more neurons and fewer 463 microglial activation in AIM2 KO than WT mice following chronic cerebral 464 hypoperfusion.

465

466

467

AIM2 KO mice are resistant to microglial activation, myelin breakdown, hippocampal neuronal loss and cognitive deficits following chronic cerebral 469 hypoperfusion

There was a lower expression of lba-1 in the cerebral cortex and hippocampus of AIM2 KO than WT mice after BCAS (Fig. 4a-f), especially in CD86 positive inflammatory microglial cells (Fig. 4c and f and Supplementary Fig. 19a-h). These results suggest a role for AIM2 in microglial activation and polarization during chronic cerebral hypoperfusion. We next investigated if AIM2 KO mice are protected against BCAS-induced myelin injury. Although sham-operated WT and AIM2 KO mice 
476 displayed no difference in protein expression and immunoreactivity of MBP, at 30 477 days after BCAS, there was lower expression and weaker immunoreactivity for MBP 478 in the cerebral cortex of WT mice than AIM2 KO mice (Fig. 4g-i). A similar difference 479 in MBP immunoreactivity between WT and AIM2 KO was seen in CA2 and CA3 480 hippocampal regions (Fig. 4j-I). As myelin integrity was healthier in AIM2 KO mice 481 following BCAS, we assessed whether these mice might be resistant to WML. After 482 BCAS, Luxol fast blue staining in WT mice indicated myelin rarefaction and vacuole 483 formation in several WM regions, but a smaller degree of damage was present in the 484 corpus callosum (paramedian or medial), caudoputamen or internal capsule regions 485 of AIM2 KO mice (Fig. 5a-e). Neuronal loss in hippocampal CA1, CA2 and CA3 486 regions was also less in AIM2 KO compared to WT mice at 15 and 30 days of 487 cerebral hypoperfusion (Fig. 5f-h). These data confirm a deleterious role of the AIM2 488 inflammasome in mediating $\mathrm{VaD}$ pathologies associated with chronic cerebral 489 hypoperfusion.

491 Finally, we tested for a role of the AIM2 inflammasome in BCAS-induced cognitive 492 decline. It was previously shown that BCAS-induced chronic cerebral hypoperfusion 493 in mice resulted in cognitive impairment such as working memory dysfunction and 494 memory loss ${ }^{29,30}$. To examine the effects of AIM2 KO against BCAS-induced 495 cognitive deficits, we conducted the Morris Water Maze and two other control tests 496 (Fig. 6a-h). To ensure that the animals were able to detect the visual cues during the 497 Morris Water Maze, a visual acuity test was conducted. All animals showed a 100\% 498 passing rate, and so proceeded to the subsequent tests. In the open field test 499 conducted to investigate explorative and locomotor ability, WT BCAS mice did not 500 have a shorter distance travelled (Fig. 6a) or a lower average speed of movement 501 (Fig. 6b) when compared to WT Sham controls, indicating no impairment of 502 locomotor ability. Similarly, there was also no significant difference in the time spent 503 and the distance travelled in the outer and inner zone among the WT mice, showing 504 no BCAS-induced anxiety effects (Fig. 6c \& d). However, it was observed that both 505 sham and BCAS AIM2 KO mice spent more time at the outer zone as compared to 506 the WT controls (Fig. 6c), indicating that AIM2 KO mice generally exhibit higher 507 levels of anxiety independent of BCAS. Several parameters were assessed in the 508 Morris Water Maze performance, including escape latency during five training days. 509 Generally, we observed a shorter time was taken to reach the platform each day 
510 across all groups. Similar daily improvement was exhibited in WT or AIM2 KO mice

511 sham controls and AIM2 KO mice following BCAS, whereas WT mice subjected to

512 BCAS took approximately twice as long (Fig. 6e). Following five days of training,

513 spatial memory was assessed whereby WT mice subjected to BCAS spent less time

514 around the platform and significantly fewer visits to the target quadrant than WT

515 controls (Fig. 6f-h). By contrast, AIM2 KO mice subjected to BCAS spent a longer

516 time around the platform and visited the target quadrant more often than WT BCAS

517 mice (Fig. 6f-h). This suggest that AIM2 KO mice exhibit better memory retention as

518 compared to WT controls under similar BCAS conditions. Thus, the behavioral data

519 indicates that BCAS-induced cognitive impairments were reduced in mice deficient in

520 AIM2, suggesting a pivotal role for the AIM2 inflammasome in mediating VaD

521 pathology associated with chronic cerebral hypoperfusion.

522

523 Discussion

524 Our findings indicate that inflammasome receptors are expressed in the cortex and

525 hippocampus where the AIM2 inflammasome is activated in response to chronic

526 cerebral hypoperfusion, leading to cellular pathology and cognitive impairment in a

527 mouse model of vascular dementia. Genetic deficiency of AIM2 expression resulted

528 in less brain inflammation, white matter injury, neuronal loss and cognitive decline

529 during cerebral hypoperfusion. These data are the first to indicate that the AIM2

530 inflammasome promotes neuronal and white matter injury following cerebral

531 hypoperfusion, identifying AIM2 as a key contributor to sterile inflammatory

532 responses in vascular dementia.

533

$534 \quad$ Inflammasomes are intracellular multiprotein complexes composed of sensors for

535 various microbial components, viral RNA and damage- or danger-associated

536 molecular patterns (DAMPs) produced during cell injury ${ }^{40,41}$. These innate immune

537 complexes are categorized according to their structural characteristics as either

538 nucleotide-binding domain-like receptors (NLRs) or absent in melanoma 2 (AIM2)-

539 like receptors ${ }^{41,42}$. These receptors oligomerize in response to activation by external

540 stimuli and then recruit the adaptor protein, apoptosis-associated speck-like protein

541 containing a caspase recruitment domain (ASC) ${ }^{43}$. Ultimately, binding of caspase-1

542 results in its cleavage and activation, and the generation of mature pro-inflammatory 
543 cytokines, IL-1 $\beta$ and IL-18, as well as pro-apoptotic cleaved caspase-3, and pro544 pyroptotic N-terminal GSDMD and GSDME ${ }^{44}$. While our study established the role of 545 AIM2 in chronic hypoperfusion-induced brain injury, it is still unknown whether other 546 inflammasomes might have a role in WML formation and neuronal cell death 547 following hypoperfusion. Although our data shows increased expression levels of 548 NLRP3 and NLRC4 in brain tissues following BCAS suggesting a potential 549 involvement of other inflammasome complexes, further studies are required to 550 establish the role of these inflammasomes in chronic hypoperfusion-induced brain 551 injury.

552

We previously demonstrated that inflammasomes contribute to neuronal cell death 554 following ischemic stroke ${ }^{7}$, in part via NF-kB and MAPK signaling ${ }^{34}$. Our present data 555 indicate that chronic cerebral hypoperfusion may also lead to increased expression 556 and activation of NLRP and AIM2 inflammasome receptors in multiple brain areas, 557 especially in the hippocampal region, which is known to be particularly vulnerable to 558 injury following mild ischemia ${ }^{45}$. Cerebral hypoperfusion in the BCAS model used 559 here is known to impact the hippocampus by mechanisms involving ionic imbalance, 560 excitotoxicity, mitochondrial dysfunction and oxidative stress ${ }^{46-48}$. I'm not sure what 561 the purpose of this sentence is.

562

563 It is important to elucidate the mechanism(s) by which the AIM2 inflammasome 564 mediates chronic hypoperfusion-induced brain injury. Here we found that the plasma 565 concentration of dsDNA progressively increased following BCAS-induced cerebral 566 hypoperfusion, consistent with the possibility that this ligand promoted AIM2567 mediated amplification of brain inflammation and injury. Thus it is plausible that AIM2 568 functions as a DAMP sensor to promote sterile inflammation in vascular dementia. 569 We found that various white matter regions exhibited injury within 7 days of BCAS, 570 consistent with the known vulnerability of white matter to hypoperfusion. Reduced 571 blood flow to the white matter has been shown to predict the clinical development of 572 WMLs within 18 months $^{49}$. We observed that AIM2 deficient animals subjected to 573 BCAS exhibited greater white matter integrity and lower WML severity scores than 574 WT mice after BCAS. Using magnetic resonance imaging, WMLs appear as white 575 matter hyperintensities (WMH) in VaD patients that arises due to demyelination and 576 axonal loss as well as microglial and endothelial activation ${ }^{50-52}$. In accordance with 
577 previous studies, we found that neuronal cell death, loss of myelin basic protein and 578 microglial activation were present in our BCAS mouse model, which were reduced in 579 AIM2 deficient mice. Our data also showed that cerebral blood flow was significantly 580 improved in AIM2 KO mice compared to WT controls following 30 days of BCAS. 581 While we do not know the precise molecular and cellular mechanisms behind this 582 increase, it is possible that reduced inflammasome activation and brain injury may 583 protect the integrity of the neurovasculature to facilitate improved blood flow to the 584 brain.

585

The sub-regions of the dorsal hippocampus, CA1, CA2 and CA3, are responsible 587 for memory encoding and retrieval ${ }^{53,54}$. Our study showed that neurons in CA2 and 588 CA3 hippocampal regions experienced elevated levels of death sooner in 589 comparison to neurons in the CA1 hippocampal regions during chronic hypoperfusion. 590 Reduced levels of BCAS-induced neuronal loss occurred in hippocampal CA2 and 591 CA3 areas of AIM2-deficient animals when compared to WT controls, which is 592 consistent with reduced inflammasome-mediated cell death in the hippocampal 593 region of AIM2 KO mice, suggesting that the AIM2 inflammasome contributed to 594 neuronal loss in the hippocampus during cerebral hypoperfusion.

The AIM2 receptor is widely expressed in the brain, with its highest levels occurring in microglia under physiological conditions ${ }^{55}$. Furthermore, specifically 598 within the cortex and hippocampus the AIM2 receptor is most abundantly expressed 599 in neurons. ${ }^{56}$. Our data are consistent with a role of AIM2 in these brain resident cells 600 and regions, but we cannot exclude circulating leukocytes as another possible cell 601 mediating the pro-inflammatory actions of AIM2 in the brain in chronic hypoperfusion. 602 Leukocytes contribute to other forms of brain injury and neurodegeneration ${ }^{57-59}$ and 603 their infiltration from the circulation would be expected in association with reduced 604 blood-brain barrier integrity and blood vessel density known to occur in 605 hypoperfusion-induced $\mathrm{VaD}^{60}$. However, in this study we have not assessed the role 606 of leukocyte infiltration and related neutrophil extracellular traps (NETs), known as 607 NETosis, as a potential source of AIM2 ligand. It was previously established that 608 NETosis and its associated extracellular dsDNA is known to contribute to the 609 pathogenesis of number of inflammatory diseases ${ }^{61}$. In addition, recent evidences 610 suggest that neutrophils use an inflammasome- and GSDMD-dependent mechanism 
611 to activate NETosis, suggesting that further studies are needed to understand the 612 role of NETosis in the pathology of $\mathrm{VaD}^{62}$.

613

614 Previous studies have recently reported that the AIM2 inflammasome contributes 615 to brain injury and chronic post-stroke cognitive impairment in mice ${ }^{63}$, and to reduced 616 neuroplasticity and spatial memory in a mouse model of $A D^{20}$. Similarly, there is also 617 evidence supporting a role for the NLRP3 inflammasome in cognitive impairment in 618 models of dementia and diabetes ${ }^{9,64}$. Inflammation is known to adversely impact 619 cognitive function, and our data are consistent with inflammasomes promoting an 620 innate immune response to mediate such an effect during cerebral hypoperfusion.

621 Moreover, we observed in our data that AIM2 KO mice generally exhibited higher 622 levels of anxiety independent of BCAS. This was reported by a previous study that 623 established that AIM2 KO mice exhibited increased anxious behaviors and reduced 624 auditory fear memory at the physiological level ${ }^{65}$.

625

626 In summary, we have provided substantial evidence that activation of the AIM2 627 inflammasome plays a key role in promoting brain inflammation, white matter lesions, 628 neuronal cell death and cognitive impairment induced by chronic cerebral 629 hypoperfusion. These effects of AIM2 involve promotion of apoptosis and pyroptosis 630 of cortical and hippocampal neurons. As inflammasome activation plays a major role 631 in a number of inflammatory diseases, there are substantial efforts to develop 632 inflammasome inhibitors. However, application of inflammasome inhibitors is limited 633 in clinical conditions as clinical trials have not yet been completed. While our study 634 establishes that the AIM2 inflammasome may therefore represent a promising 635 therapeutic target for attenuating cognitive impairment in $\mathrm{VaD}$, further research is 636 warranted to develop specific AIM2 inhibitors to test their effect in experimental and 637 clinical studies. 


\section{Acknowledgements}

647 We thank Professor Jenny P. Ting (University of North Carolina, Chapel Hill, Chapel 648 Hill, NC, USA) for providing the AIM2 deficient mice. Supplementary Figure 1 and 649 Supplementary Figure 9a in this article was created using BioRender.

650

\section{Funding}

652 This work was supported by the National Medical Research Council Research Grants 653 (NMRC-CBRG-0102/2016 and NMRC/OFIRG/0036/2017), Singapore and the startup 654 fund to TVA from La Trobe University, Melbourne, Australia.

655

\section{Author Contributions}

657 Study Conception \& Design: T.V.A., L.P., and D.Y.F.; Experiment or Data Collection: 658 T.V.A., L.P., P.W., H.M.L., S.L.F., S.W.K., V.R., S.S., D.Y.F., R.I.V., and N.P.; Data 659 Analysis: L.P., P.W., V.R., S.S., D.Y.F., and T.V.A.; Data Interpretation: L.P., T.V.A., 660 and D.Y.F; Writing-Manuscript Preparation and intellectual input: T.V.A., L.P., D.Y.F., 661 M.B.K., G.R.D., C.G.S., D.C.H., D.G.J., M.K.P.L., C.L.H.C., and L.H.K.L.; 662 Supervision \& Administration: T.V.A., D.Y.F., M.K.P.L., and C.L.H.C.

663

\section{Conflicts Of Interest}

665 The authors declare that the research was conducted in the absence of any 666 commercial or financial relationships that could be construed as a potential conflict of 667 interest.

668

669

670

671

672

673

674

675

676

677 


\section{REFERENCES}

680

681

682

683

684

685

686

687

688

689

690

691

692

693

694

695

696

697

698

699

700

701

702

703

704

705

1) Wolters FJ, Ikram MA. Epidemiology of Vascular Dementia. Arterioscler Thromb Vasc Biol 2019; 39:1542-1549.

2) Van der Flier WM, Skoog I, Schneider JA, Pantoni L, Mok V, Chen CLH, et al. Vascular cognitive impairment. Nat Rev Dis Primers 2018; 4: 18003.

3) Alber J, Alladi S, Bae HJ, Barton DA, Beckett LA, Bell JM, et al. White matter hyperintensities in vascular contributions to cognitive impairment and dementia (VCID): Knowledge gaps and opportunities. Alzheimers Dement (N Y) 2019; 5: 107-117.

4) Schmitz M, Hermann P, Oikonomou P, Stoeck K, Ebert E, Poliakova T, et al. Cytokine profiles and the role of cellular prion protein in patients with vascular dementia and vascular encephalopathy. Neurobiol Aging 2015; 36: 2597-2606.

5) Zhou $Y$, Zhang J, Wang $L$, Chen $Y$, Wan $Y$, He $Y$, et al. Interleukin-1 $\beta$ impedes oligodendrocyte progenitor cell recruitment and white matter repair following chronic cerebral hypoperfusion. Brain Behav Immun 2017; 60: 93-105.

6) Fann DY, Lee SY, Manzanero S, Chunduri P, Sobey CG, Arumugam TV. Pathogenesis of acute stroke and the role of inflammasomes. Ageing Res Rev 2013; 12: 941-966.

7) Fann DY, Lee SY, Manzanero S, Tang SC, Gelderblom M, Chunduri P, et al. Intravenous immunoglobulin suppresses NLRP1 and NLRP3 inflammasomemediated neuronal death in ischemic stroke. Cell Death Dis 2013; 4: e790.

8) Gordon R, Albornoz EA, Christie DC, Langley MR, Kumar V, Mantovani S, et al. Inflammasome inhibition prevents a-synuclein pathology and dopaminergic neurodegeneration in mice. Sci Transl Med 2018; 10: 465.

9) Heneka MT, Kummer MP, Stutz A, Delekate A, Schwartz S, Vieira-Saecker A, et al. NLRP3 is activated in Alzheimer's disease and contributes to pathology in APP/PS1 mice. Nature 2013; 493: 674-678.

10) Johann S, Heitzer M, Kanagaratnam M, Goswami A, Rizo T, Weis J, et al. NLRP3 inflammasome is expressed by astrocytes in the SOD1 mouse model of ALS and in human sporadic ALS patients. Glia 2015; 63: 2260-2273.

11) Poh L, Kang SW, Baik SH, Ng GYQ, She DT, Balaganapathy $P$, et al. Evidence that NLRC4 inflammasome mediates apoptotic and pyroptotic microglial death following ischemic stroke. Brain Behav Immun 2019; 75: 34-47.

12) Fritsch M, Günther SD, Schwarzer R, Albert MC, Schorn F, Werthenbach JP, et al. Caspase-8 is the molecular switch for apoptosis, necroptosis and pyroptosis. Nature 2019; 575: 683-687.

13) Ball DP, Taabazuing CY, Griswold AR, Orth EL, Rao SD, Kotliar IB, et al. Caspase-1 interdomain linker cleavage is required for pyroptosis. Life Sci Alliance 2020; 3: 3.

14) Yuan B, Zhou XM, You ZQ, Xu WD, Fan JM, Chen SJ, et al. Inhibition of AIM2 inflammasome activation alleviates GSDMD-induced pyroptosis in early brain injury after subarachnoid haemorrhage. Cell Death Dis 2020; 11: 76.

15) Liu X, Zhang Z, Ruan J, Pan Y, Magupalli VG, Wu H, et al. Inflammasomeactivated gasdermin $D$ causes pyroptosis by forming membrane pores. Nature 2016; 535: 153-158.

16) Rogers C, Erkes DA, Nardone A, Aplin AE, Fernandes-Alnemri T, Alnemri ES. Gasdermin pores permeabilize mitochondria to augment caspase-3 activation during apoptosis and inflammasome activation. Nat Commun 2019; 10: 1689. 
728

729

730

731

732

733

734

735

736

737

738

739

740

741

742

743

744

745

746

747

748

749

750

751

752

753

754

755

756

757

758

759

760

761

762

763

764

765

766

767

768

769

770

771

772

773

774

775

776

17) Kayagaki N, Stowe IB, Lee BL, O'Rourke K, Anderson K, Warming S, et al. Caspase-11 cleaves gasdermin $D$ for non-canonical inflammasome signalling. Nature 2015; 526: 666-671.

18) McKenzie BA, Mamik MK, Saito LB, Boghozian R, Monaco MC, Major EO, et al. Caspase-1 inhibition prevents glial inflammasome activation and pyroptosis in models of multiple sclerosis. Proc Natl Acad Sci USA 2018; 115: E6065-E6074.

19) Dempsey C, Rubio Araiz A, Bryson KJ, Finucane O, Larkin C, et al. Inhibiting the NLRP3 inflammasome with MCC950 promotes non-phlogistic clearance of amyloid- $\beta$ and cognitive function in APP/PS1 mice. Brain Behav Immun 2017; 61: 306-316.

20) Chen J, Shu S, Chen Y, Liu Z, Yu L, Yang L, et al. AlM2 deletion promotes neuroplasticity and spatial memory of mice. Brain Res Bull 2019; 152: 85-94.

21) Briard $B$, Place DE, Kanneganti TD. DNA Sensing in the Innate Immune Response. Physiology (Bethesda) 2020; 35: 112-124.

22) Hornung V, Ablasser A, Charrel-Dennis M, Bauernfeind F, Horvath G, Caffrey DR, et al. AlM2 recognizes cytosolic dsDNA and forms a caspase-1-activating inflammasome with ASC. Nature 2009; 458: 514-518.

23) Jakobs C, Perner S, Hornung V. AIM2 Drives Joint Inflammation in a Self-DNA Triggered Model of Chronic Polyarthritis. PLoS One 2015; 10: e0131702.

24) Xiao TS. The nucleic acid-sensing inflammasomes. Immunol Rev 2015; 265: 103-111.

25) Paganelli R, Di lorio A, Patricelli L, Ripani F, Sparvieri E, Faricelli $R$, et al. Proinflammatory cytokines in sera of elderly patients with dementia: levels in vascular injury are higher than those of mild-moderate Alzheimer's disease patients. Exp Gerontol 2002; 37: 257-263.

26) Yasutake C, Kuroda K, Yanagawa T, Okamura T, Yoneda H. Serum BDNF, TNFalpha and IL-1beta levels in dementia patients: comparison between Alzheimer's disease and vascular dementia. Eur Arch Psychiatry Clin Neurosci 2006; 256: 402-406.

27) Zuliani G, Ranzini M, Guerra G, Rossi L, Munari MR, Zurlo A, et al. Plasma cytokines profile in older subjects with late onset Alzheimer's disease or vascular dementia. J Psychiatr Res 2007; 41: 686-693.

28) Mulugeta E, Molina-Holgado F, Elliott MS, Hortobagyi T, Perry R, Kalaria RN, et al. Inflammatory mediators in the frontal lobe of patients with mixed and vascular dementia. Dement Geriatr Cogn Disord 2008; 25: 278-286.

29) Shibata $M$, Ohtani $R$, Ihara $M$, Tomimoto $H$. White matter lesions and glial activation in a novel mouse model of chronic cerebral hypoperfusion. Stroke 2004; 35: 2598-2603.

30) Shibata M, Yamasaki N, Miyakawa T, Kalaria RN, Fujita $Y$, Ohtani R, et al. Selective impairment of working memory in a mouse model of chronic cerebral hypoperfusion. Stroke 2007; 38: 2826-2832.

31) Washida K, Hattori $Y$, Ihara M. Animal Models of Chronic Cerebral Hypoperfusion: From Mouse to Primate. Int J Mol Sci. 2019; 20: 6176.

32) Wilson JE, Petrucelli AS, Chen L, Koblansky AA, Truax AD, Oyama $Y$, et al. Inflammasome-independent role of AIM2 in suppressing colon tumorigenesis via DNA-PK and Akt. Nat Med 2015; 21: 906-913.

33) Khan MB, Hafez S, Hoda MN, Baban B, Wagner J, Awad ME, et al. Chronic Remote Ischemic Conditioning Is Cerebroprotective and Induces Vascular Remodeling in a VCID Model. TransI Stroke Res 2018; 9: 51-63. 
782

783

784

785

786

787

788

789

790

791

792

793

794

795

796

797

798

799

800

801

802

803

804

805

806

807

808

809

810

811

812

813

814

815

816

817

818

819

820

821

822

823

824

825

34) Fann DY, Lim YA, Cheng YL, Lok KZ, Chunduri $P$, Baik SH, et al. Evidence that NF-kB and MAPK Signaling Promotes NLRP Inflammasome Activation in Neurons Following Ischemic Stroke. Mol Neurobiol 2018; 55: 1082-1096.

35) Nishio K, Ihara M, Yamasaki N, Kalaria RN, Maki T, Fujita Y, et al. A Mouse Model Characterizing Features of Vascular Dementia With Hippocampal Atrophy. Stroke 2010; 41: 1278-1284.

36) Thaung C, Arnold K, Jackson IJ, Coffey PJ. Presence of visual head tracking differentiates normal sighted from retinal degenerate mice. Neurosci Lett. 2002; 325: 21-24.

37) Mitchiner JC, Pinto LH, Vanable JW Jr. Visually evoked eye movements in the mouse (Mus musculus). Vision Res. 1976; 16: 1169-1171.

38) Wong P, Chang CC, Marx CE, Caron MG, Wetsel WC, Zhang X. Pregnenolone rescues schizophrenia-like behavior in dopamine transporter knockout mice. PLoS One. 2012; 7: e51455.

39) Madar R, Rotter A, Waldman Ben-Asher H, Mughal MR, Arumugam TV, Wood $\mathrm{WH} 3^{\text {rd }}$, et al. Postnatal TLR2 activation impairs learning and memory in adulthood. Brain Behav Immun. 2015; 48: 301-312.

40) Feldman N, Rotter-Maskowitz A, Okun E. DAMPs as mediators of sterile inflammation in aging-related pathologies. Ageing Res Rev 2015; 24: 29-39.

41) Lamkanfi $M$, Dixit VM. Inflammasomes and their roles in health and disease. Annu Rev Cell Dev Biol 2012; 28: 137-161.

42) Schroder K, Tschopp J. The inflammasomes. Cell 2010; 140, 821-832.

43) Lu A, Magupalli VG, Ruan J, Yin Q, Atianand MK, Vos MR, et al. Unified polymerization mechanism for the assembly of ASC-dependent inflammasomes. Cell 2014; 156: 1193-1206.

44) Xue Y, Enosi Tuipulotu D, Tan WH, Kay C, Man SM. Emerging Activators and Regulators of Inflammasomes and Pyroptosis. Trends Immunol 2019; 40: 10351052.

45) Shaw K, Bell L, Boyd K, Grijseels DM, Clarke D, Bonnar O, et al. Hippocampus has lower oxygenation and weaker control of brain blood flow than cortex, due to microvascular differences. BioRxiv, 2019; 835728.

46) Almeida A, Allen KL, Bates TE, Clark JB. Effect of Reperfusion Following Cerebral Ischaemia on the Activity of the Mitochondrial Respiratory Chain in the Gerbil Brain. Journal of Neurochemistry 2002; 65: 1698-1703.

47) Belov Kirdajova D, Kriska J, Tureckova J, Anderova M. Ischemia-Triggered Glutamate Excitotoxicity From the Perspective of Glial Cells. Frontiers in Cellular Neuroscience, 2020; 14: 51.

48) Heuser D, Guggenberger H. Ionic Changes in Brain Ischaemia and Alterations Produced by Drugs. British Journal of Anaesthesia 1985; 57: 23-33.

49) Bernbaum M, Menon BK, Fick G, Smith EE, Goyal M, Frayne R, et al. Reduced blood flow in normal white matter predicts development of leukoaraiosis. Journal of Cerebral Blood Flow \& Metabolism 2015; 35: 1610.

50) Fazekas F, Kleinert R, Offenbacher H, Schmidt R, Kleinert G, Payer F, et al. Pathologic correlates of incidental MRI white matter signal hyperintensities. Neurology 1993; 43, 1683-1689.

51) Fernando MS, O'Brien JT, Perry RH, English P, Forster G, McMeekin W, et al. Comparison of the pathology of cerebral white matter with post-mortem magnetic resonance imaging $(\mathrm{MRI})$ in the elderly brain. Neuropathology and Applied Neurobiology 2004; 30: 385-395. 
826

827

828

829

830

831

832

833

834

835

836

837

838

839

840

841

842

843

844

845

846

847

848

849

850

851

852

853

854

855

856

857

858

859

860

861

862

863

864

865

866

867

868

869

870

871

872

873

874

875
52) Wardlaw JM, Valdés Hernández MC, Muñoz $\square$ Maniega S. What are White Matter Hyperintensities Made of? Journal of the American Heart Association 2015; 4: 001140.

53) Mankin EA, Diehl GW, Sparks FT, Leutgeb S, Leutgeb JK. Hippocampal CA2 Activity Patterns Change over Time to a Larger Extent than between Spatial Contexts. Neuron 2015; 85: 190-201.

54) Mao H, Elkin BS, Genthikatti VV, Morrison B III, Yang KH. Why Is CA3 More Vulnerable Than CA1 in Experimental Models of Controlled Cortical ImpactInduced Brain Injury? Journal of Neurotrauma 2013; 30: 1521.

55) Zhang Y, Chen K, Sloan SA, Bennett ML, Scholze AR, O'Keeffe S, et al. An RNA-Sequencing Transcriptome and Splicing Database of Glia, Neurons, and Vascular Cells of the Cerebral Cortex. The Journal of Neuroscience 2014; 34: 11929.

56) Wu PJ, Liu HY, Huang TN, Hsueh YP. AIM 2 inflammasomes regulate neuronal morphology and influence anxiety and memory in mice. Scientific Reports 2016; 6: 32405.

57) Brochard V, Combadière B, Prigent $A$, Laouar $Y$, Perrin A, Beray-Berthat $V$, et al. Infiltration of $C D 4+$ lymphocytes into the brain contributes to neurodegeneration in a mouse model of Parkinson disease. J Clin Invest 2009; 119: 182-192.

58) Chu HX, Kim HA, Lee S, Moore JP, Chan CT, Vinh A, et al. Immune cell infiltration in malignant middle cerebral artery infarction: comparison with transient cerebral ischemia. J Cereb Blood Flow Metab 2014; 34: 450-459.

59) Zhang SR, Piepke M, Chu HX, Broughton BR, Shim R, Wong CH, et al. IL-33 modulates inflammatory brain injury but exacerbates systemic immunosuppression following ischemic stroke. JCI Insight 2018; 3: 18.

60) Roberts JM, Maniskas ME, Bix GJ. Bilateral carotid artery stenosis causes unexpected early changes in brain extracellular matrix and blood-brain barrier integrity in mice. PLoS One 2018; 13: e0195765.

61) Liu J, Yang D, Wang X, Zhu Z, Wang T, Ma A, et al. Neutrophil extracellular traps and dsDNA predict outcomes among patients with ST-elevation myocardial infarction. Sci Rep. 2019; 9: 11599.

62) Chen KW, Monteleone M, Boucher D, Sollberger G, Ramnath D, Condon ND, et al. Noncanonical inflammasome signaling elicits gasdermin D-dependent neutrophil extracellular traps. Sci Immunol. 2018; 3: eaar6676.

63) Kim H, Seo JS, Lee SY, Ha KT, Choi BT, Shin Yl, et al. AIM2 inflammasome contributes to brain injury and chronic post-stroke cognitive impairment in mice. Brain Behav Immun 2020; 87: 765-776.

64) Ward R, Li W, Abdul Y, Jackson L, Dong G, Jamil S, et al. NLRP3 inflammasome inhibition with MCC950 improves diabetes-mediated cognitive impairment and vasoneuronal remodeling after ischemia. Pharmacol Res 2019; 142: 237-250.

65) Wu PJ, Liu HY, Huang TN, Hsueh YP. AIM 2 inflammasomes regulate neuronal morphology and influence anxiety and memory in mice. Sci Rep. 2016; 6: 32405. 


\section{FIGURE LEGENDS}

877 Figure 1: Effect of chronic cerebral hypoperfusion on inflammasome activation 878 and cell death in the cerebral cortex and hippocampus over time following

879 BCAS. (a \& b), representative immunoblots and quantification illustrating increased 880 levels of activated inflammasome effector proteins such as cleaved caspase-1 (p20), $881-8$, and -11 , and maturation of downstream effector targets, IL-1 $\beta$ and IL-18, in the 882 cerebral cortex. (c \& d), representative immunoblots and quantification illustrating 883 increased levels of activated inflammasome effector proteins such as cleaved 884 caspase-1 (p20, p33), -8, and -11, and maturation of downstream effector targets, IL$8851 \beta$ and IL-18, in the hippocampus. (e \& f), representative immunoblots and 886 quantification illustrating increases in the expression of apoptotic marker cleaved 887 caspase-3 and pyroptotic marker N-terminal GSDMD in the cerebral cortex. (g \& h), 888 representative immunoblots and quantification illustrating increases in the expression 889 of apoptotic marker cleaved caspase-3 and pyroptotic marker N-terminal GSDMD in 890 the hippocampus. $\beta$-actin was used as a loading control. Data are represented as 891 mean \pm S.E.M. $n=6-7$ mice in each experimental group. ${ }^{*} \mathrm{P}<0.05$ compared with 892 Sham; ${ }^{* *} P<0.01$ compared with Sham; ${ }^{* *} P<0.001$ compared with Sham. 893 Abbreviations: BCAS, bilateral common carotid artery stenosis; Cl, cleaved; FL, full 894 length; NT, N-terminal; GSDMD, gasdermin D; GSDME, gasdermin E.

897 Figure 2: Effect of chronic cerebral hypoperfusion on the levels of glial 898 activation, white matter integrity and hippocampal neuronal density in the 899 cerebral cortex and hippocampus following BCAS. (a), representative 900 immunoblots and quantification illustrating increased microglial activation due to 901 increased levels of Iba-1 in the cerebral cortex over time following BCAS. Data are 902 represented as mean \pm S.E.M. $n=6-7$ mice in each experimental group. $\beta$-actin was 903 used as a loading control. ${ }^{*} \mathrm{P}<0.05$ compared with Sham. (b), representative 904 immunofluorescence analysis of lba-1 and GFAP in the cerebral cortex provide 905 supporting evidence of microglial activation following BCAS. Magnification x 100.

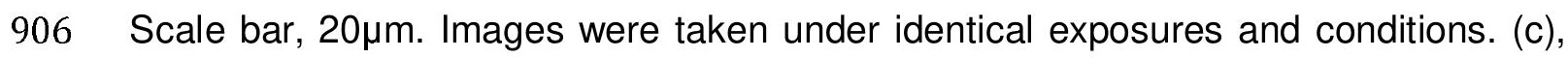
907 representative immunoblots and quantification illustrating increased astroglia 908 activation due to increased levels of GFAP in the hippocampus over time following 909 BCAS. Data are represented as mean \pm S.E.M. $n=6-7$ mice in each experimental 
910 group. $\beta$-actin was used as a loading control. ${ }^{*} \mathrm{P}<0.05$ compared with Sham;

$911{ }^{* *} \mathrm{P}<0.01$ compared with Sham; ${ }^{* *} \mathrm{P}<0.001$ compared with Sham. (d), representative

912 immunofluorescence analysis of Iba-1 and GFAP in the hippocampus provide

913 supporting evidence of astroglia activation following BCAS. Magnification x 100.

914 Scale bar, 20 $\mu \mathrm{m}$. Images were taken under identical exposures and conditions. (e \&

$915 \mathrm{f})$, representative merged immunofluorescence images of DAPI, CD86 and CD206

916 co-localized within microglia (Iba-1 positive) in the cerebral cortex and hippocampus

917 of WT controls following BCAS. Representative merged immunofluorescence images

918 illustrate the activation of CD86 (M1) and CD206 (M2) positive microglia in the

919 cerebral cortex (e) and hippocampus (f) following BCAS. ( $g$ \& h), representative

920 merged immunofluorescence images of DAPI, Complement C3 (C3) and S100A10

921 within astrocytes (GFAP positive) in the cerebral cortex and hippocampus of WT

922 controls following BCAS. Other than colocalization of S100A10 (A2) within astrocytes

923 in cerebral cortex (g), no substantial co-localization of C3 (A1) was observed in the

924 cerebral cortex and hippocampus following BCAS. This illustrates activation of A2

925 astrocytes in the cerebral cortex (g) upon BCAS. Zoom Magnification x 100. Scale

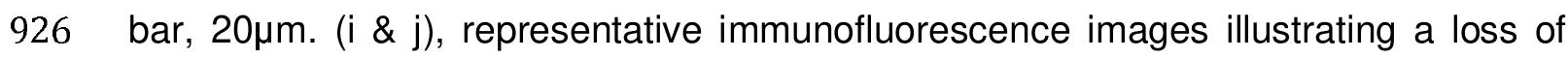

927 myelin due to decreased levels of MBP immunoreactivity in the cerebral cortex and

928 hippocampus following BCAS. ( $\mathrm{k}$ \& I), representative immunofluorescence images

929 illustrating a loss of neurons due to decreased levels of MAP2 immunoreactivity in

930 the cerebral cortex and hippocampus following BCAS. Magnification x 20. Scale bar,

$931120 \mu \mathrm{m}$. ( $\mathrm{m} \& \mathrm{n})$, representative Luxol fast blue stained images and quantification

932 illustrating disruption of white matter integrity due to increased myelin rarefaction and

933 white matter lesion formation in the corpus callosum (paramedian), corpus callosum

934 (medial), caudoputamen, internal capsule and optic tract over time following BCAS.

935 The severity of white matter disruption was graded accordingly: Grade $0=$ no

936 disruption; Grade 1 = disarrangement of nerve fibers; Grade 2 = formation of marked

937 vacuoles; Grade 3 = disappearance of myelinated fibers. Images were taken under

938 identical exposures and conditions. (o-r), representative crystal violet images and

939 quantification illustrating loss of Nissl positively stained neurons in hippocampal CA1,

940 CA2 and CA3 regions over time following BCAS. Data are represented as mean \pm

941 S.E.M. $\mathrm{n}=5-7$ mice in each experimental group. ${ }^{*} \mathrm{P}<0.05$ compared with Sham;

$942{ }^{* *} \mathrm{P}<0.01$ compared with Sham; ${ }^{* *} \mathrm{P}<0.001$ compared with Sham. Magnification $\times 60$.

943 Scale bar, $20 \mu \mathrm{m}$. Images were taken under identical exposures and conditions. 
944 Abbreviations: BCAS, bilateral common carotid artery stenosis; CD86, cluster of 945 differentiation 86; CD206, cluster of differentiation 206; C3, complement component 946 3; GFAP, glial fibrillary acidic protein; Iba-1, ionized calcium binding adaptor 947 molecule-1; MBP, myelin basic protein; MAP2, microtubule-associated protein 2; 948 S100A10, S100 calcium-binding protein A10.

949

950 Figure 3: Effect of chronic cerebral hypoperfusion on inflammasome-mediated 951 programmed cell death in AIM2 KO mice following BCAS. (a), quantification 952 illustrating increased serum concentrations of the AIM2 receptor ligand, cell free 953 double stranded DNA (dsDNA), over time in wild-type mice following BCAS. Data are 954 represented as mean \pm S.E.M. $n=5-7$ mice in each experimental group. ${ }^{*} \mathrm{P}<0.05$ 955 compared with Sham. (b-e), representative immunoblots and quantification 956 illustrating suppression of inflammasome activation due to decreased expression of 957 cleaved caspase-1 (p20) at Day 15 and 30, and maturation of IL-1 $\beta$ cytokine 958 production at Day 30 in the cerebral cortex (b \& c) and hippocampus ( $d$ \& e) of AIM2 959 KO mice compared to WT controls following BCAS. (f-i), representative immunoblots 960 and quantification illustrating attenuation of apoptotic and pyroptotic cell death due to 961 decreased expression of cleaved caspase-3 and GSDMD-NT, respectively, at Day 96230 in the cerebral cortex ( $f$ \& $g$ ) and hippocampus ( $h$ \& i) of AIM2 KO mice compared 963 to WT controls following BCAS. $\beta$-actin was used as a loading control. Data are 964 represented as mean \pm S.E.M. $n=5-8$ mice in each experimental group. ${ }^{*} \mathrm{P}<0.05$ 965 compared with WT Sham; ${ }^{* *} \mathrm{P}<0.01$ compared with WT Sham; ${ }^{* *} \mathrm{P}<0.001$ compared 966 with WT Sham. ${ }^{+} \mathrm{P}<0.05$ compared with WT BCAS; ${ }^{++} \mathrm{P}<0.01$ compared with WT 967 BCAS; ${ }^{+++} \mathrm{P}<0.001$ compared with WT BCAS. (j \& $\mathrm{k}$ ), representative merged 968 immunofluorescence images of DAPI, cleaved caspase-1 p10 (CC1), cleaved 969 caspase-3 (CC3) and GSDMD co-localized within neurons (MAP2 positive), microglia 970 (Iba-1 positive), oligodendrocytes (OLIG2 positive) and endothelial cells (PECAM-1 971 positive) in the cerebral cortex and hippocampus of WT controls following BCAS. No 972 substantial co-localization of cleaved caspase-1 p10, cleaved caspase-3 and 973 GSDMD was observed in astrocytes (GFAP positive) in the cerebral cortex and 974 hippocampus of WT controls following BCAS. (j \& k), representative merged 975 immunofluorescence images illustrate a reduction in inflammasome activation, and 976 apoptotic and pyroptotic cell death due to decreased expression levels of cleaved 977 caspase-1, cleaved caspase-3 and GSDMD, respectively, in both neurons and 
978 microglia in the cerebral cortex (j) and hippocampus (k) in AIM2 KO mice compared 979 to WT controls following BCAS. Zoom Magnification x 100. Scale bar, 20 $\square$ m. 980 Images were taken under identical exposures and conditions. Abbreviations: BCAS, 981 bilateral common carotid artery stenosis; Cl, cleaved; FL, full length; NT, N-terminal; 982 WT, wild-type; KO, knock out; CC1, cleaved caspase-1; CC3, cleaved caspase-3; 983 GSDMD, Gasdermin D; MAP2, microtubule-associated protein 2; lba-1, ionized 984 calcium binding adaptor molecule-1; OLIG2, oligodendrocyte transcription factor 2; 985 GFAP, glial fibrillary acidic protein; PECAM-1, platelet endothelial cell adhesion 986 molecule-1.

987

988 Figure 4: Effect of chronic cerebral hypoperfusion on glial activation and 989 myelin expression in the cerebral cortex and hippocampus of AIM2 KO mice 990 following BCAS. ( $a$ \& b, d \& e), representative immunoblots and quantification 991 illustrating resistance to microglial activation due to decreased expression of lba-1 in 992 the cerebral cortex and hippocampus of AIM2 KO mice compared to WT controls 993 following BCAS. No effect on astroglia activation was observed as the expression of 994 GFAP in the cerebral cortex and hippocampus remained unchanged in AIM2 KO 995 mice compared to WT controls following BCAS. $\beta$-actin was used as a loading control. 996 Data are represented as mean \pm S.E.M. $n=6-8$ mice in each experimental group. $997{ }^{*} \mathrm{P}<0.05$ compared with WT Sham; ${ }^{*} \mathrm{P}<0.01$ compared with WT Sham. $+\mathrm{P}<0.05$ 998 compared with WT BCAS; $++\mathrm{P}<0.01$ compared with WT BCAS; $+++\mathrm{P}<0.001$ 999 compared with WT BCAS. (c \& f), representative merged immunofluorescence 1000 images of DAPI, CD86 and CD206 co-localized within microglia (lba-1 positive) in the 1001 cerebral cortex and hippocampus of WT controls following BCAS. Representative 1002 merged immunofluorescence images illustrate a reduction in M1 (CD86 positive) and 1003 M2 (CD206 positive) microglial activation due to decreased expression levels of 1004 CD86 and CD206 respectively, in the cerebral cortex (c) and hippocampus (f) in 1005 AIM2 KO mice compared to WT controls following BCAS. (c \& f), representative 1006 merged immunofluorescence images of DAPI, Complement C3 (C3) and S100A10 1007 within astrocytes (GFAP positive) in the cerebral cortex and hippocampus of WT 1008 controls following BCAS. Other than colocalization of S100A10 (A2) within astrocytes 1009 in the cerebral cortex (c), no substantial co-localization of $C 3$ (A1) was observed in 1010 the cerebral cortex (c) and hippocampus ( $f$ ) of following BCAS. Representative 1011 merged immunofluorescence images illustrate a reduction in A2 (S100A10 positive) 
1012 astrocytes activation due to decreased expression levels of S100A10 in the cerebral 1013 cortex (c) in AIM2 KO mice compared to WT controls following BCAS. (g \& h, j \& k),

1014 representative immunoblots and quantification illustrating resistance to myelin loss 1015 due to increased expression of MBP in the cerebral cortex (g) and hippocampus (j) in 1016 AIM2 KO mice compared to WT controls following BCAS. $\beta$-actin was used as a 1017 loading control. Data are represented as mean \pm S.E.M. $n=5-7$ mice in each 1018 experimental group. ${ }^{*} \mathrm{P}<0.05$ compared with WT Sham; $+\mathrm{P}<0.05$ compared with WT 1019 BCAS; ++P<0.01 compared with WT BCAS ( $\mathrm{i}$ \& I), representative 1020 immunofluorescence images illustrating resistance to myelin loss due to increased 1021 MBP immunoreactivity in the cerebral cortex (i), and hippocampal CA1, CA2 and 1022 CA3 regions (I) in AIM2 KO mice compared to WT controls following BCAS.

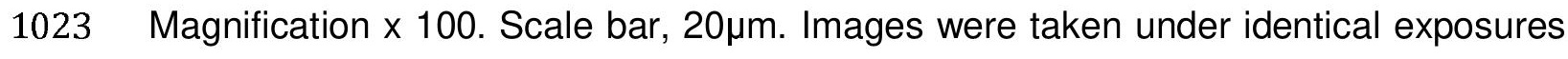
1024 and conditions. Abbreviations: BCAS, bilateral common carotid artery stenosis; CD86, 1025 cluster of differentiation 86; CD206, cluster of differentiation 206; C3, complement 1026 component 3; GFAP, glial fibrillary acidic protein; lba-1, ionized calcium binding 1027 adaptor molecule-1; KO, knock out; S100A10, S100 calcium-binding protein A10.

1028

1029 Figure 5: Effect of chronic cerebral hypoperfusion on cortical white matter 1030 integrity and hippocampal neuronal density in AIM2 KO mice following BCAS.

1031 (a-e), representative Luxol fast blue stained images and quantification illustrating 1032 preserved white matter integrity due to decreased myelin rarefaction and white 1033 matter lesion formation in the corpus callosum (paramedian), corpus callosum 1034 (medial), caudoputamen and internal capsule in AIM2 KO mice compared with WT 1035 controls following BCAS. No significant difference in white matter integrity was 1036 observed in the optic tract between AIM2 KO mice compared with WT controls 1037 following 30 days of BCAS. The severity of white matter disruption was graded 1038 accordingly: Grade 0 = no disruption; Grade 1 = disarrangement of nerve fibres; 1039 Grade 2 = formation of marked vacuoles; Grade 3 = disappearance of myelinated 1040 fibres. (f-h), representative crystal violet images and quantification illustrating 1041 increased Nissl positively stained neurons in hippocampal CA1, CA2 and CA3 1042 regions in AIM2 KO mice compared to WT controls following BCAS. Data are 1043 represented as mean \pm S.E.M. $\mathrm{n}=5-7$ mice in each experimental group. ${ }^{*} \mathrm{P}<0.05$ 1044 compared with WT Sham; ${ }^{* *} \mathrm{P}<0.01$ compared with WT Sham; ${ }^{* *} \mathrm{P}<0.001$ compared 1045 with WT Sham. ${ }^{+} \mathrm{P}<0.05$ compared with WT BCAS; ${ }^{++} \mathrm{P}<0.01$ compared with WT 
1046 BCAS; ${ }^{+++} \mathrm{P}<0.001$ compared with WT BCAS. Magnification x 60. Scale bar, $20 \square \mu \mathrm{m}$.

1047 Images were taken under identical exposures and conditions. Abbreviations: BCAS, 1048 bilateral common carotid artery stenosis; WT, wild type; KO, knock out; WML, white 1049 matter lesion.

1050

1051

1052 Figure 6: Effect of chronic cerebral hypoperfusion on explorative locomotive 1053 behavior and spatial memory in AIM2 KO mice following BCAS. (a-d), 1054 quantifications illustrating an open field test conducted on all mice to examine the 1055 locomotor ability and level of anxiety by measuring the total distance travelled (mm) 1056 and average speed of movement $(\mathrm{mm} / \mathrm{s})$; total time $(\mathrm{sec})$ and distance travelled $(\mathrm{mm})$ 1057 in the outer and inner zone of the open field. It was shown that WT BCAS mice did 1058 not have a shorter distance travelled $(\mathrm{mm})(\mathrm{a})$ and a lower average speed $(\mathrm{mm} / \mathrm{s})$ of 1059 movement (b) when compared to WT Sham controls, indicating no impairment of 1060 locomotor ability. Similarly, there are also no significant difference in the time spent 1061 and distance travelled in the outer and inner zone among WT mice, showing no 1062 BCAS-induced anxiety effect (c \& d). However, it was observed that in both sham 1063 and BCAS AIM2 KO mice more time was spent at the outer zone when compared to 1064 WT controls (c), indicating that AIM2 KO mice generally exhibit higher levels of 1065 anxiety independent of BCAS. (e-h), quantifications illustrating a Morris water maze 1066 test conducted on all mice to examine spatial learning and memory by measuring the 1067 escape latency (sec), time spent around the platform on probe day (sec), number of 1068 visits in the target quadrant and time spent in target quadrant (sec). (e), in general, a 1069 reduction in escape latency ( $\mathrm{sec}$ ) was observed in all mice across the training period. 1070 Specifically, WT Sham, AIM2 KO Sham and AIM2 KO BCAS groups displayed a 1071 trend with a shorter escape latency and steeper declining gradient, which reflected 1072 an improved learning ability when compared to the WT BCAS group. (f-h), in general, 1073 the AIM2 KO BCAS group displayed a trend with a longer time spent around the 1074 platform on probe day and a significantly higher number of visits and time spent in 1075 the target quadrant, which reflected retention of spatial memory when compared to 1076 the WT BCAS group. Data are represented as mean \pm S.E.M. $n=9-11$ mice in each 1077 experimental group. ${ }^{*} \mathrm{P}<0.05$ compared with WT Sham. \#P<0.05 compared with WT 1078 BCAS; \#\#P<0.01 compared with WT BCAS; \#\#\#P<0.001 compared with WT BCAS. 
bioRxiv preprint doi: https://doi.org/10.1101/2020.06.05.135228; this version posted November 21, 2020. The copyright holder for this preprint (which was not certified by peer review) is the author/funder. All rights reserved. No reuse allowed without permission.

1079 Abbreviations: BCAS, bilateral common carotid artery stenosis; WT, wild type; KO, 1080 knock out.

1081 


\section{Cerebral Cortex}

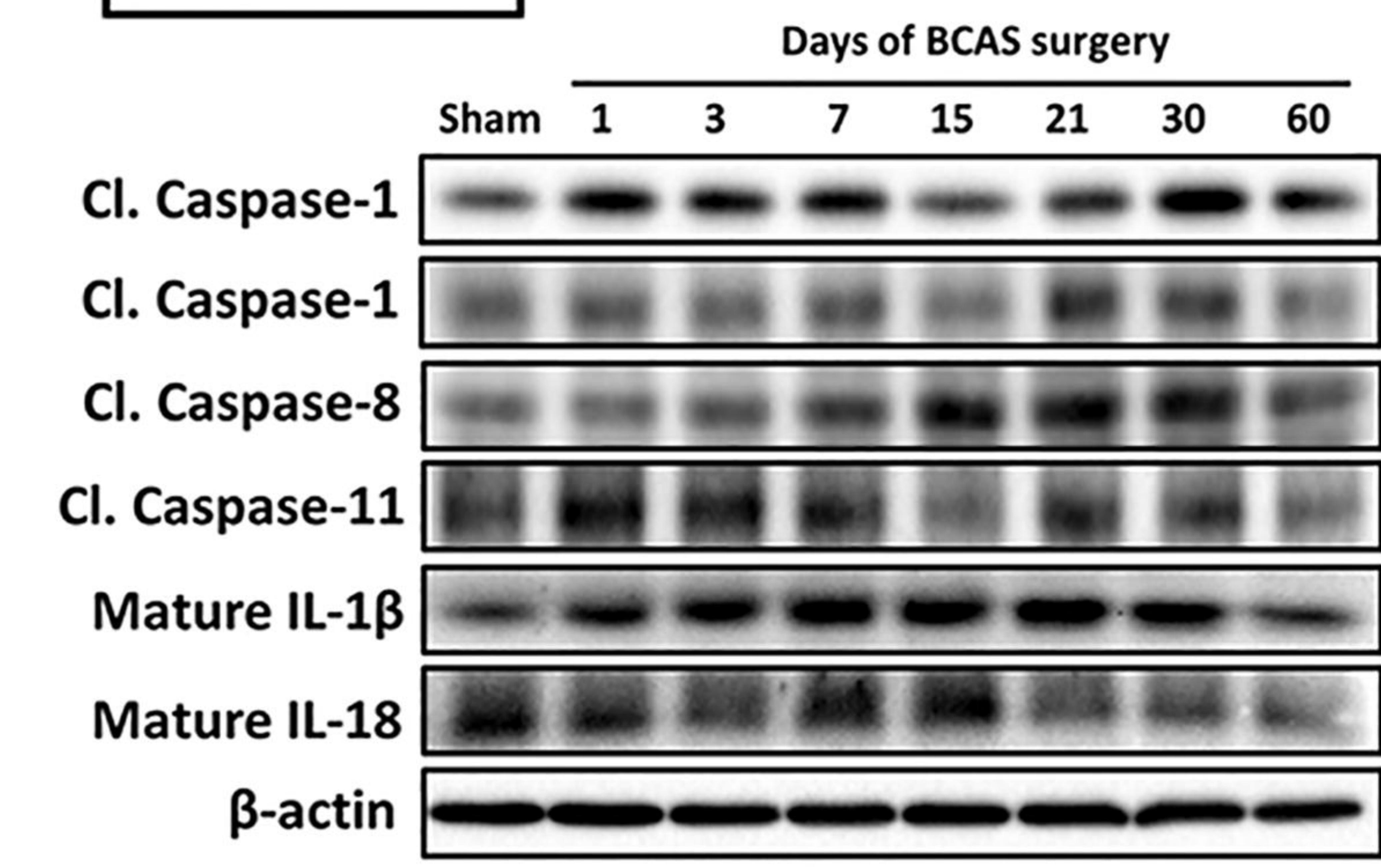

\section{Hippocampus}

\begin{tabular}{rlllllll}
\hline & \multicolumn{8}{c}{ Days of BCAS surgery } \\
\cline { 2 - 8 } Sham & 1 & 3 & 7 & 15 & 21 & 30 & 60
\end{tabular}

Cl. Caspase-1 - - - - - $20 \mathrm{kDa}$

Cl. Caspase-1 33 kDa

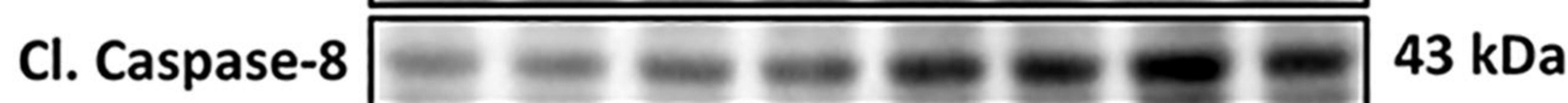

Cl. Caspase-11 32 kDa

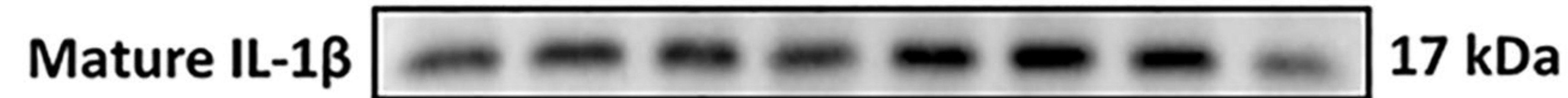

Mature IL-18 18 kDa

$\beta$-actin
$42 \mathrm{kDa}$

b

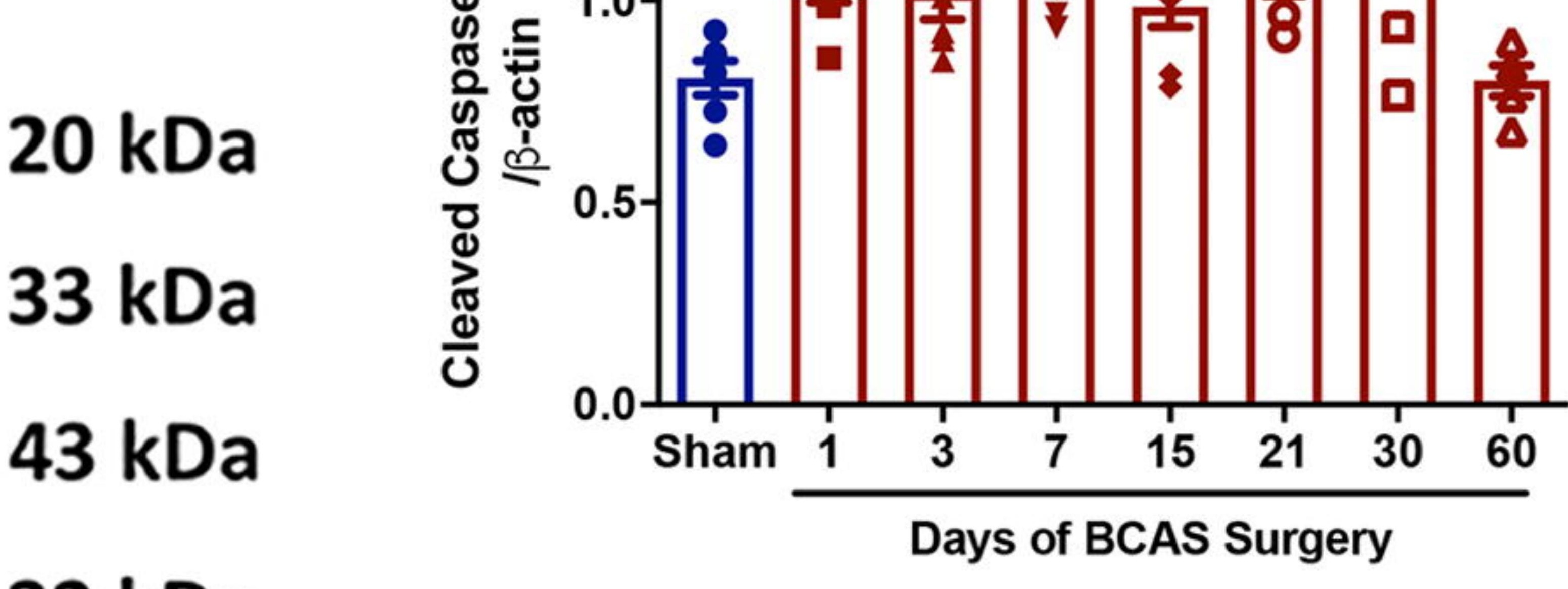

$32 \mathrm{kDa}$

$17 \mathrm{kDa}$

$42 \mathrm{kDa}$

d
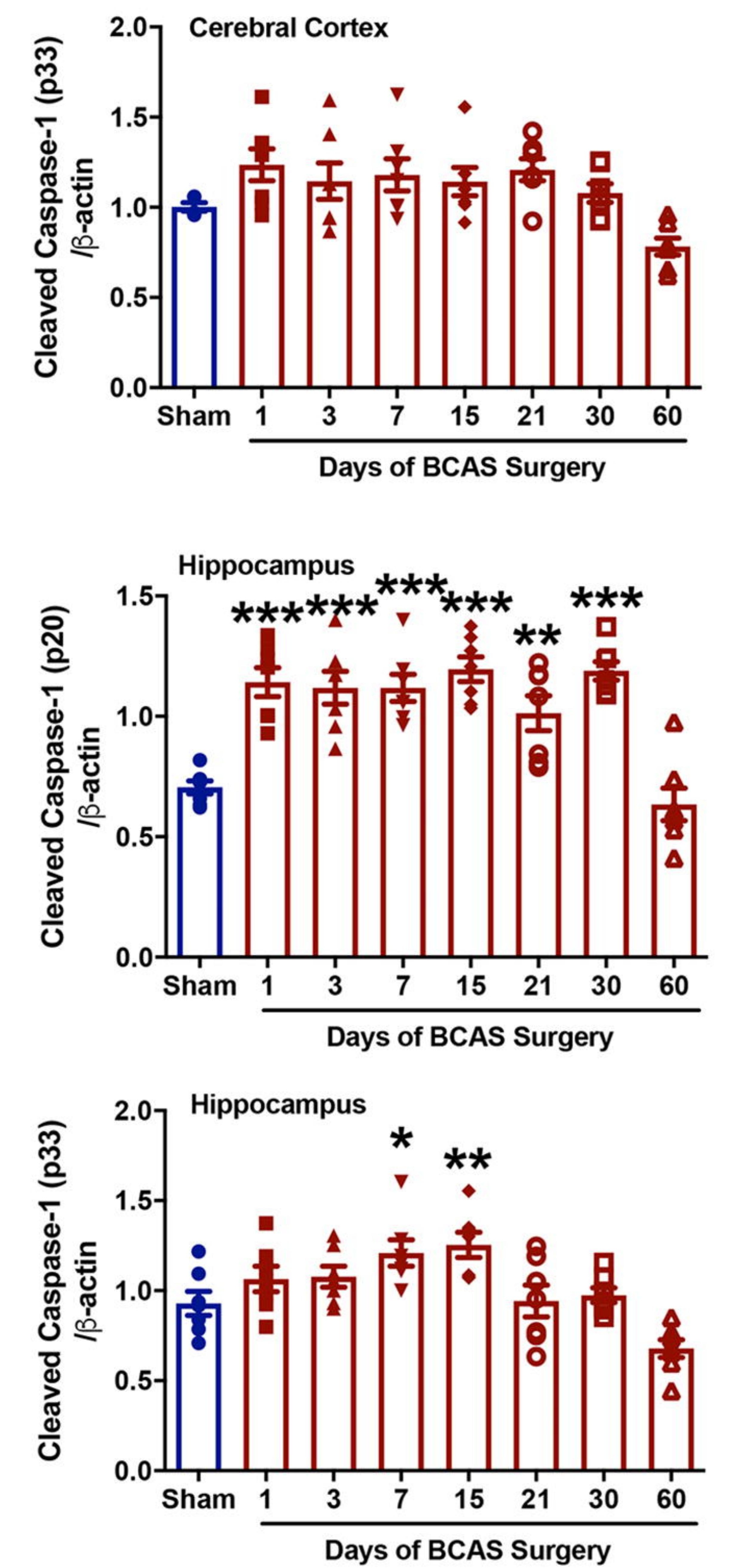
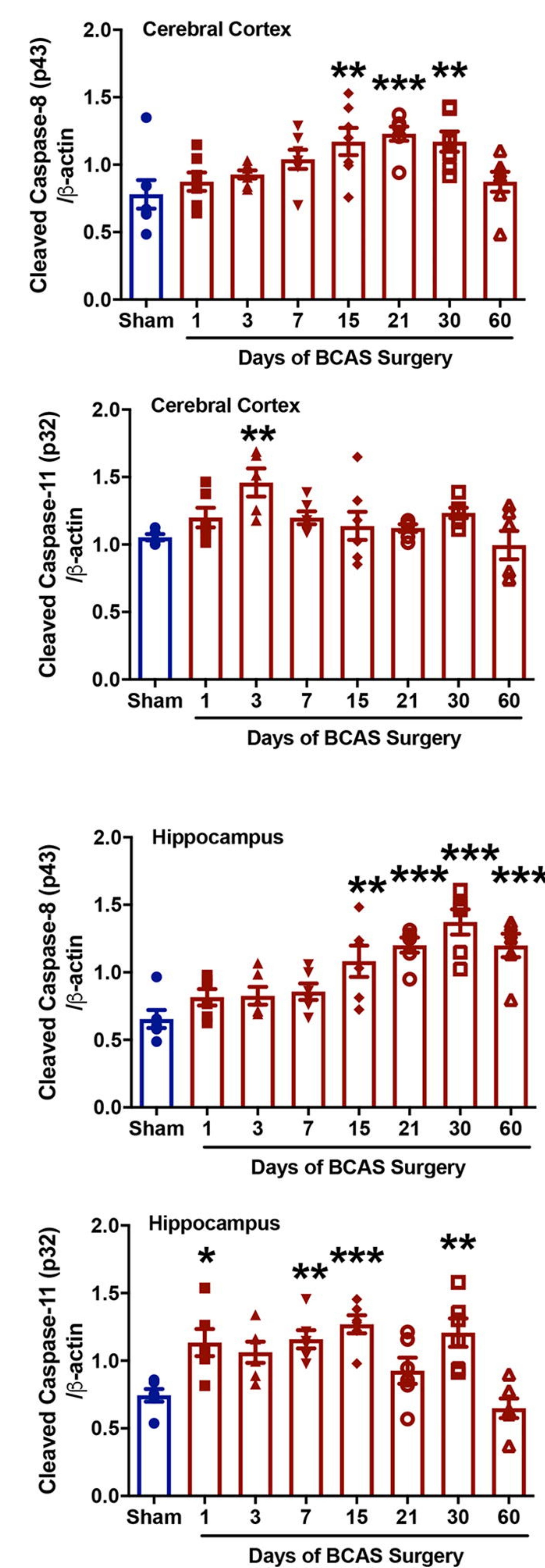
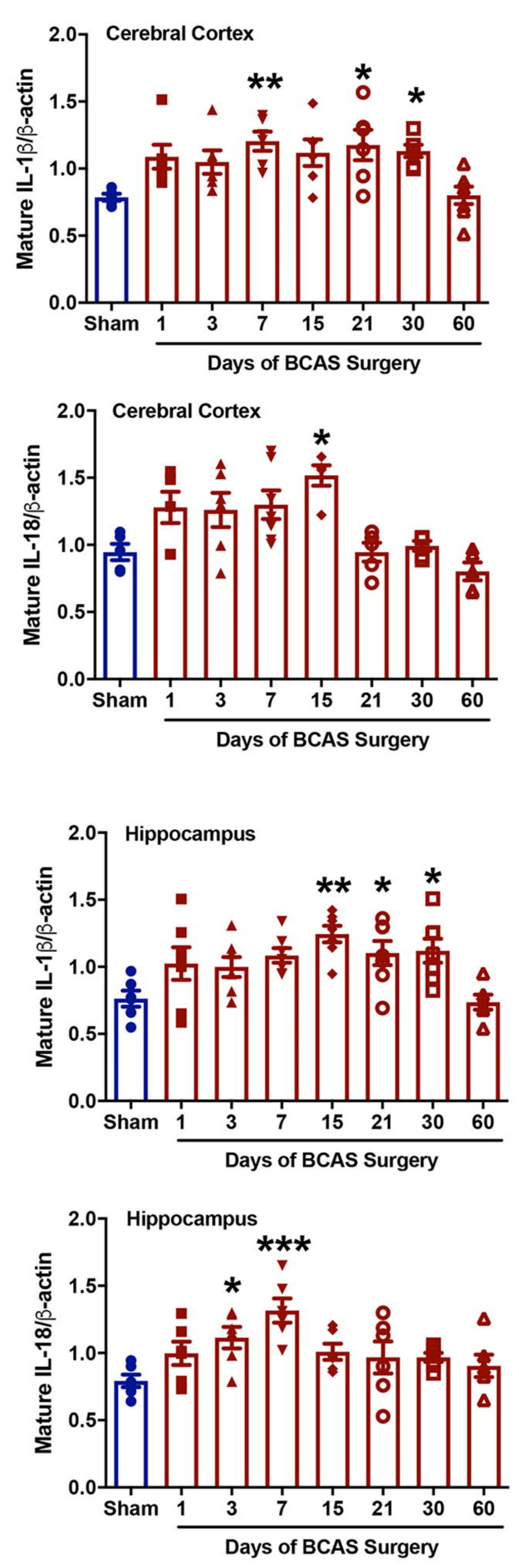

e

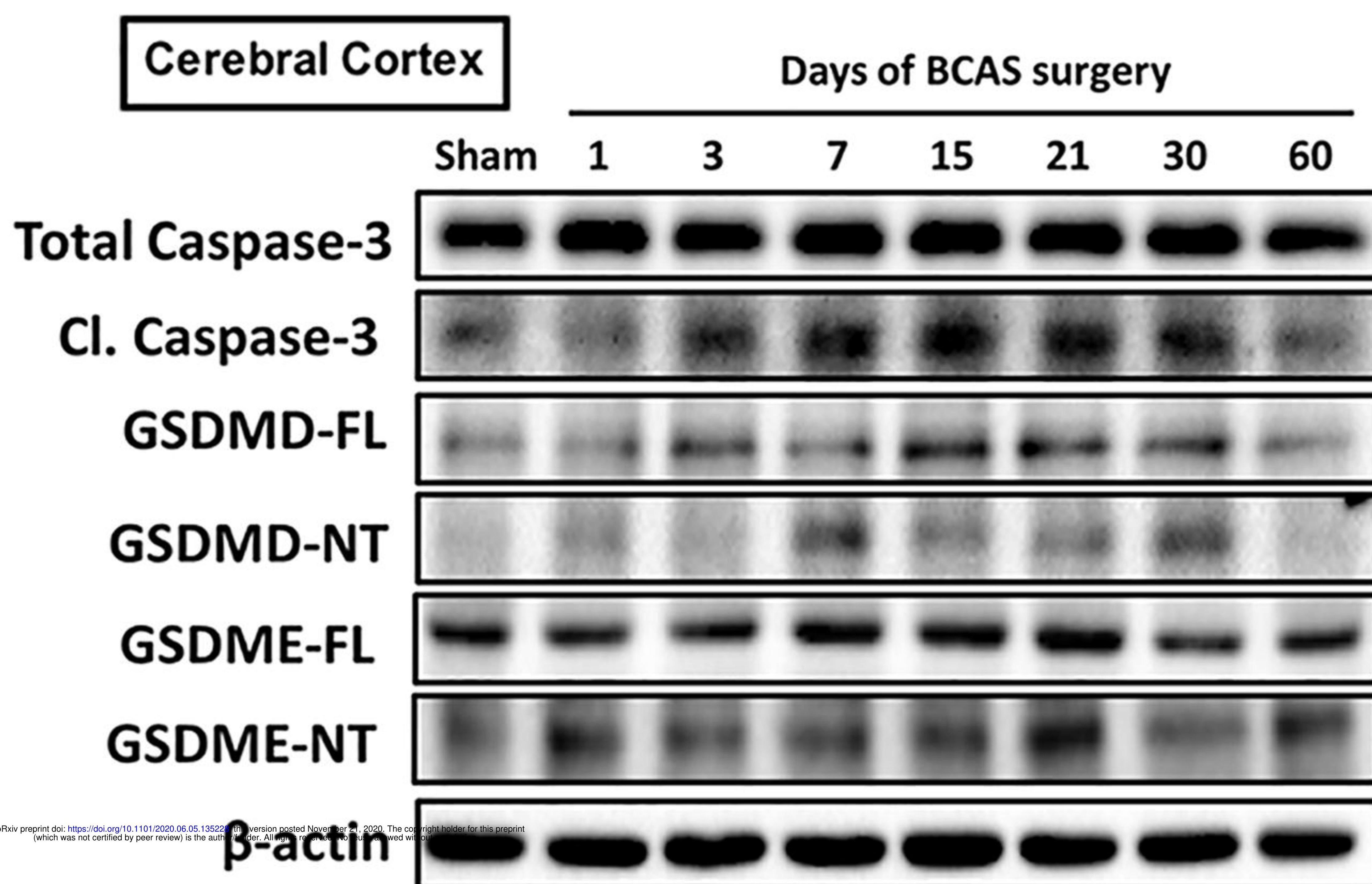

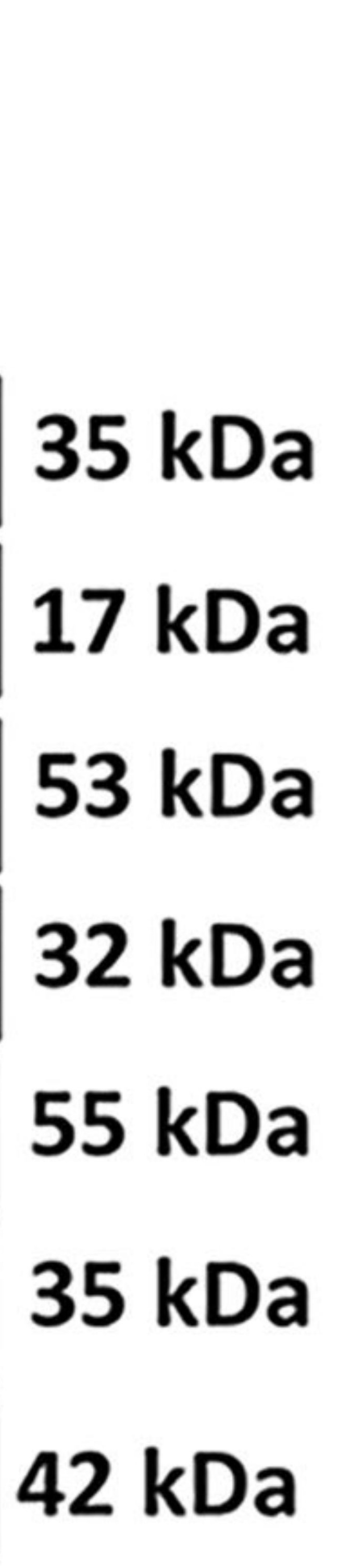

g

Hippocampus

Days of BCAS surgery

Total Caspase-3 3

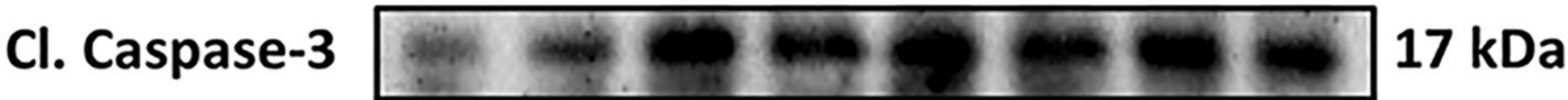

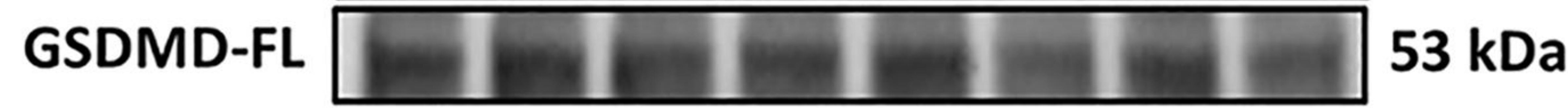

GSDMD-NT W $32 \mathrm{kDa}$

GSDME-FL $=-m 55 \mathrm{kDa}$

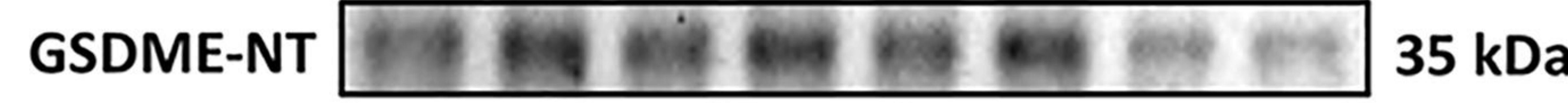

$\beta$-actin $=-m \mathrm{kDa}$

h
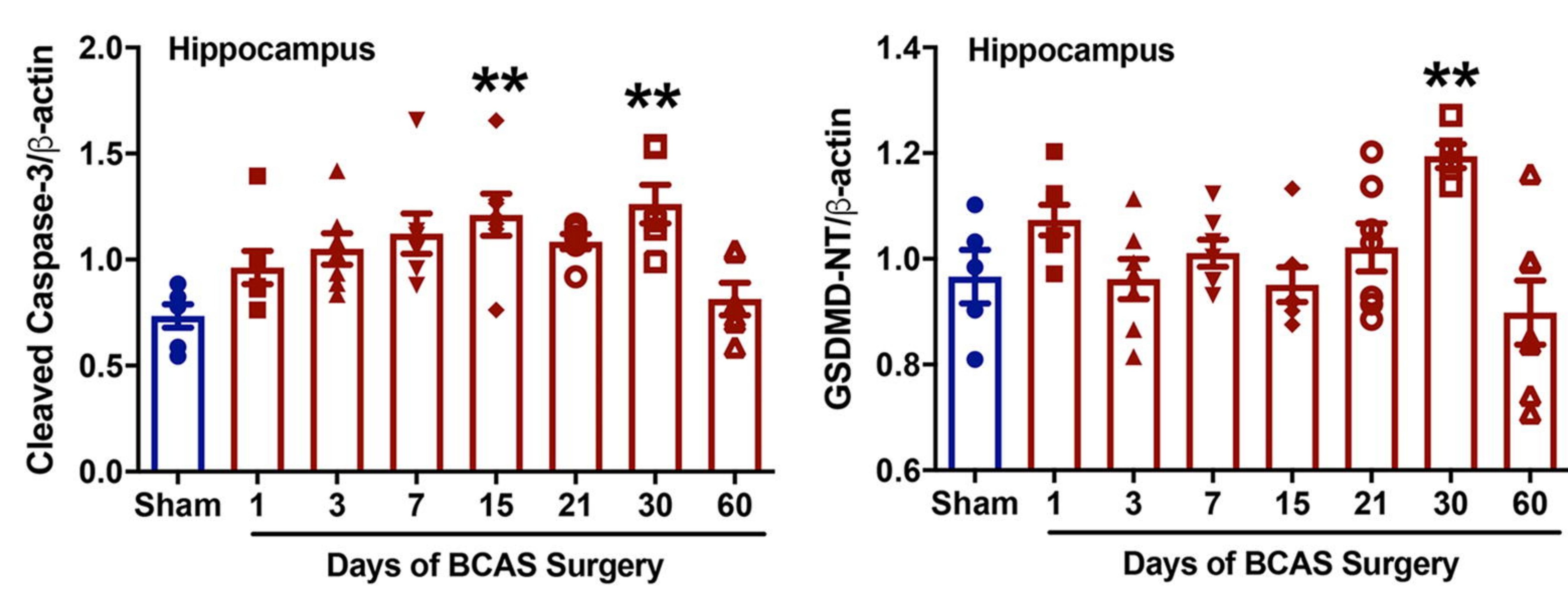

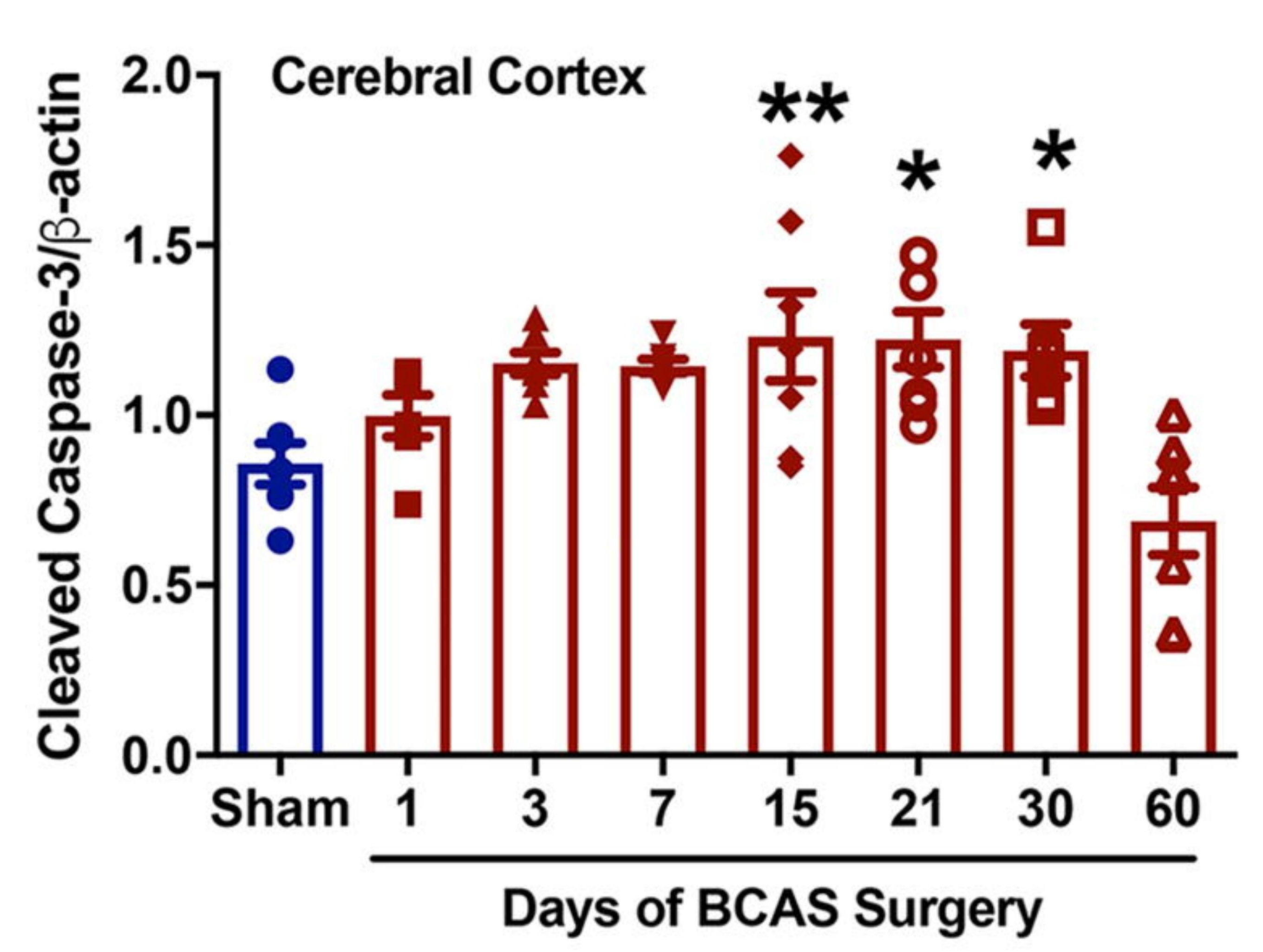

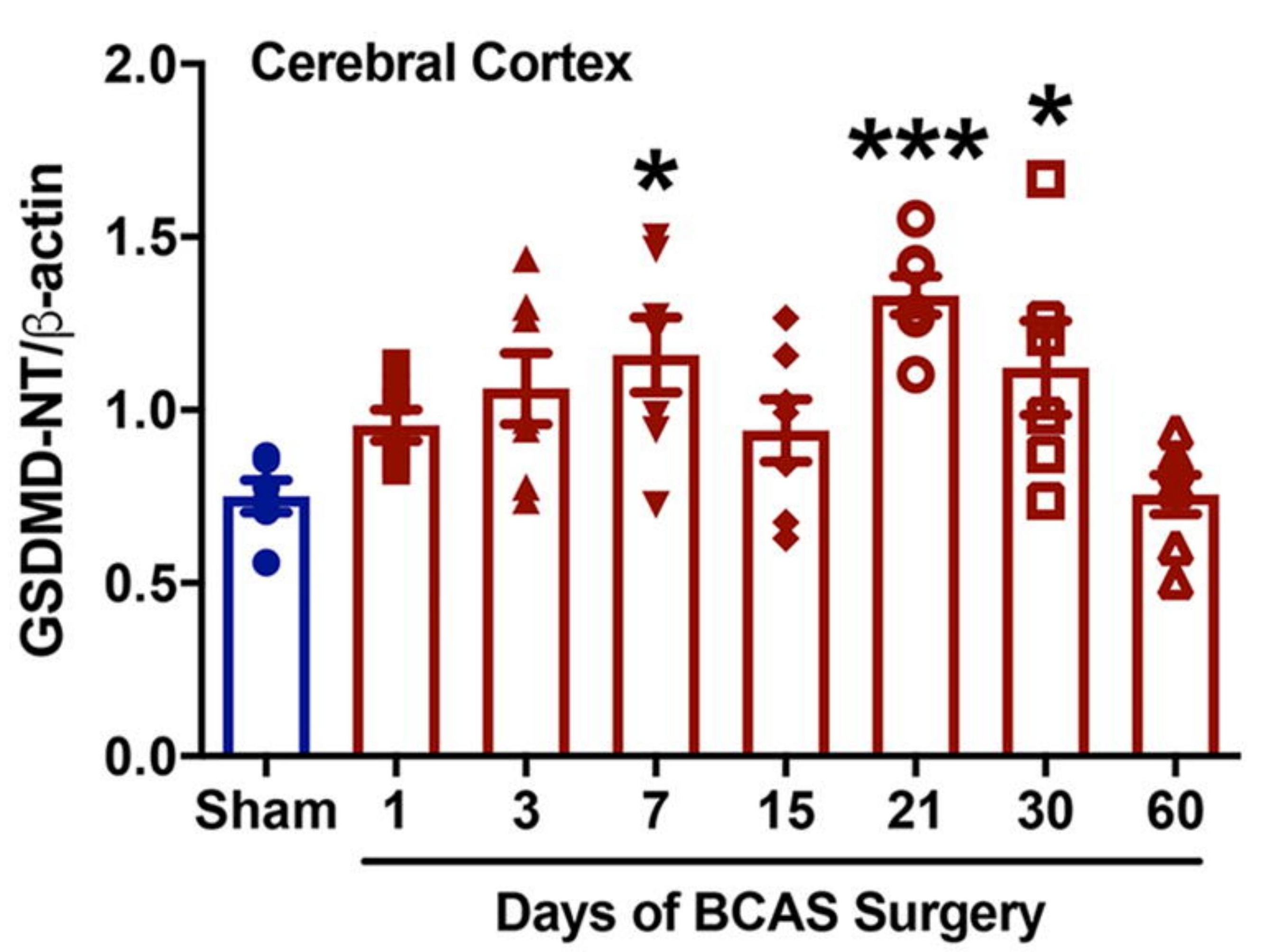




\section{$\mathbf{a}$}

a

b

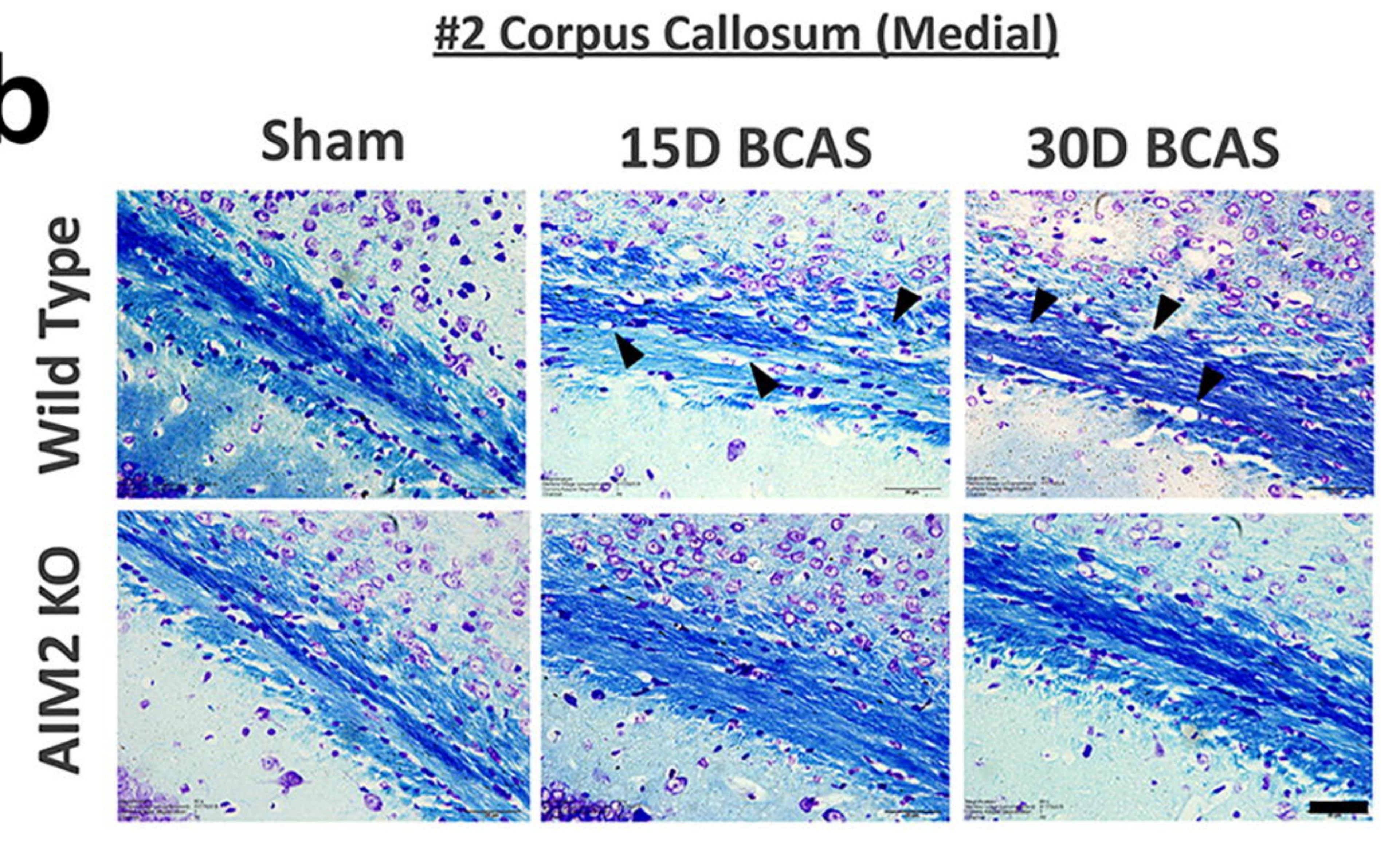

\#3 Caudoputamen

C

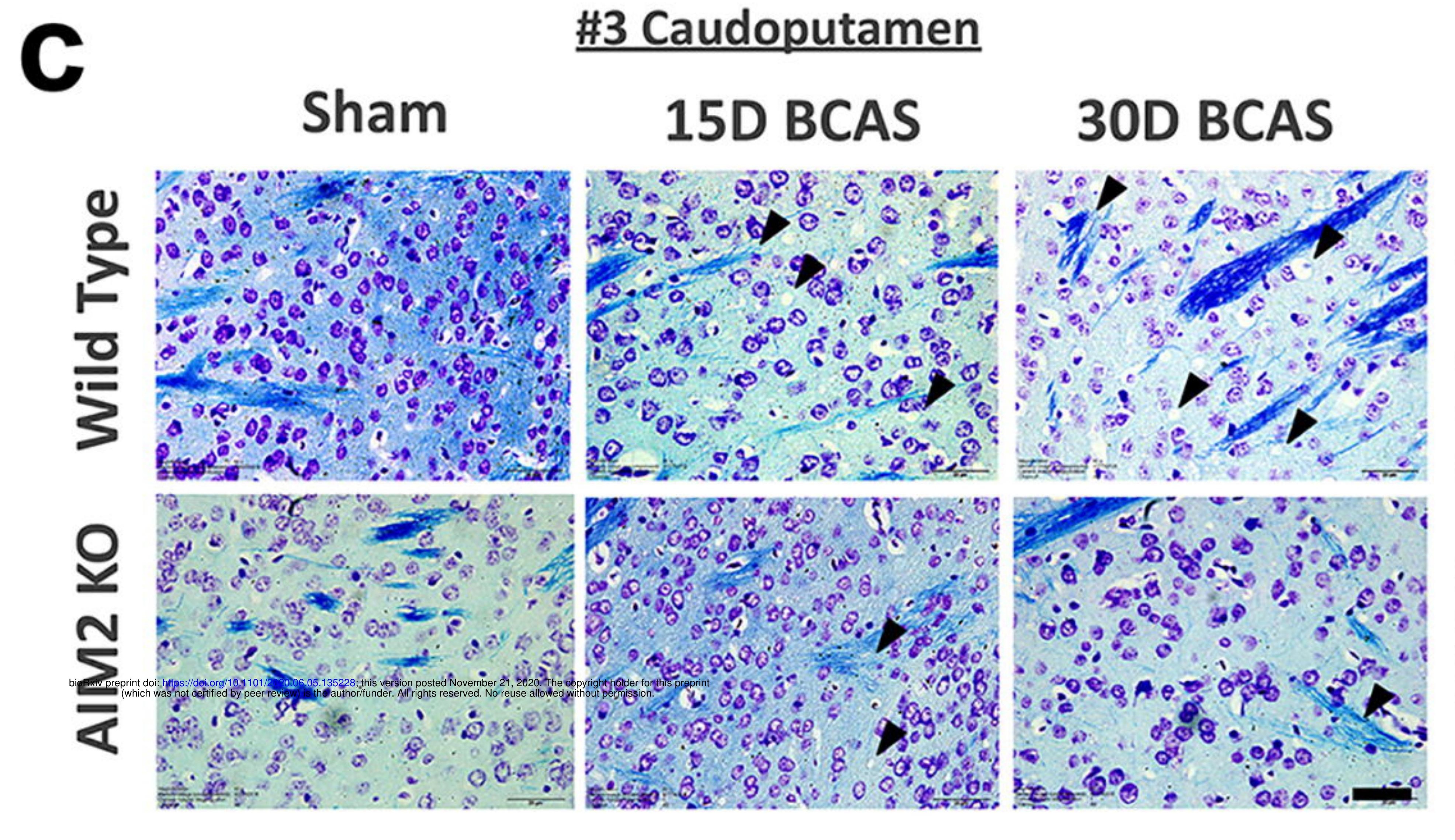

\#4 Internal Capsule

d

d

\#1 Corpus Callosum (Paramedian)

Sham 15D BCAS

30D BCAS

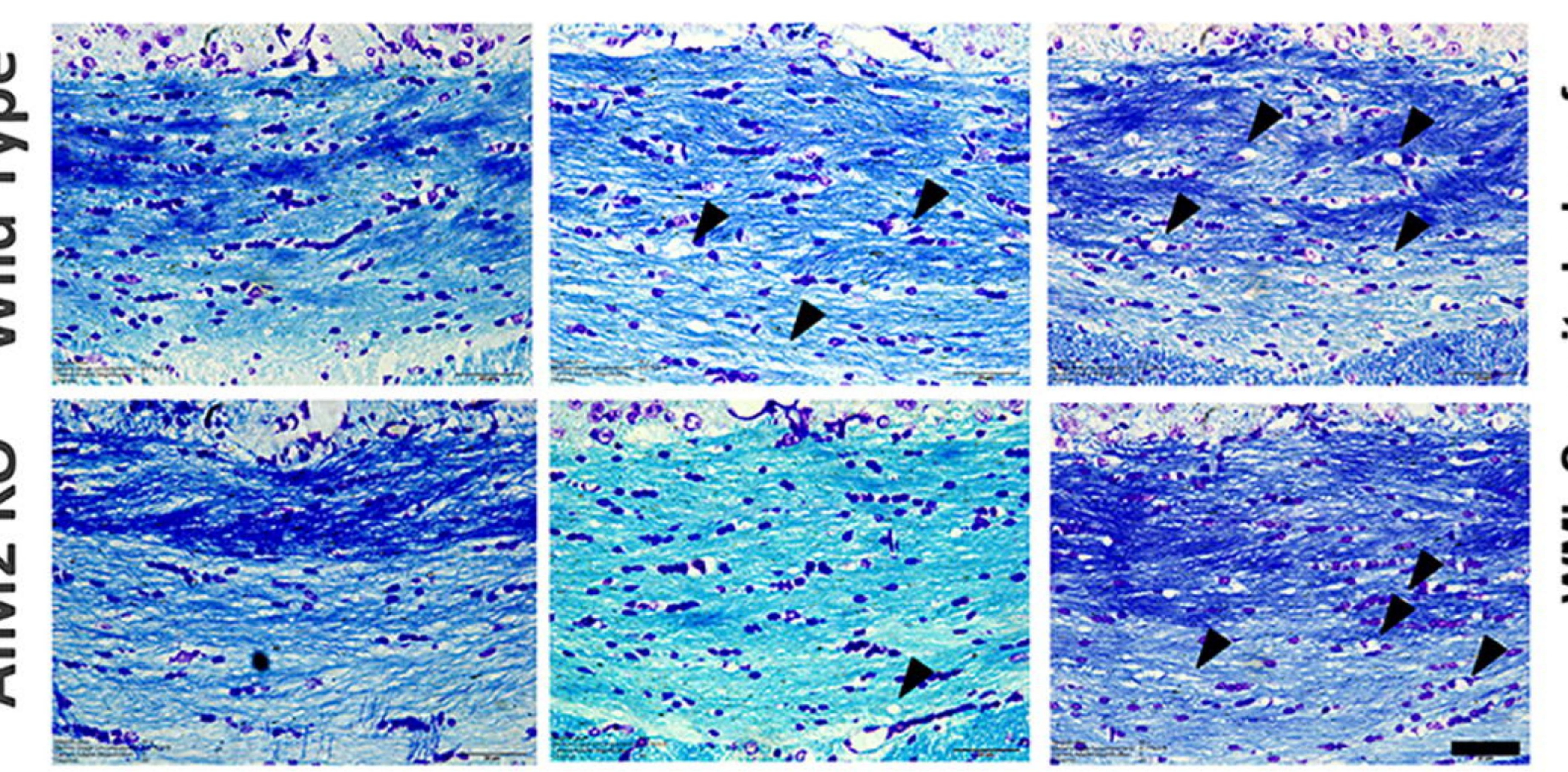

2 Corpus Callosum (Medial)

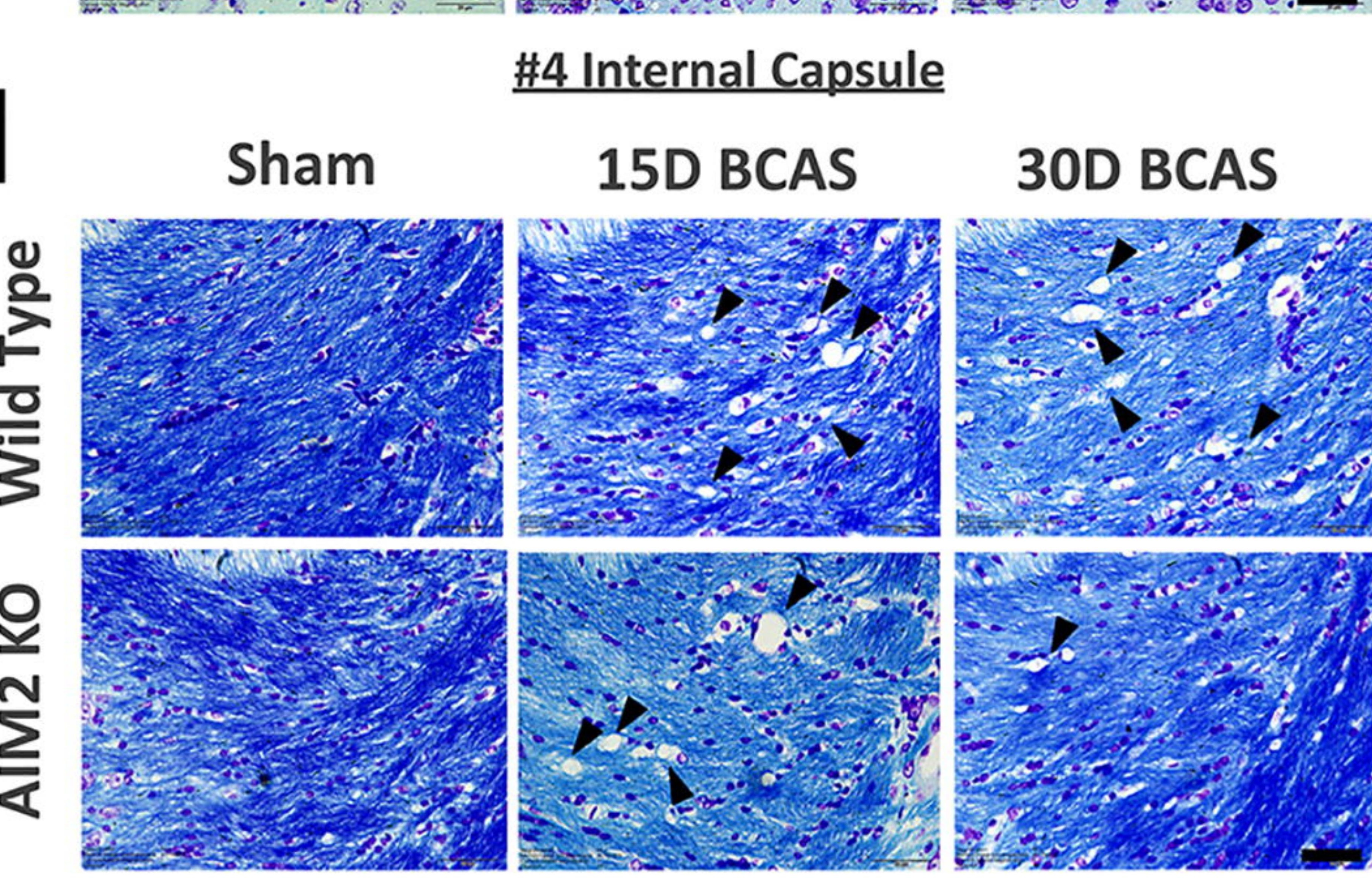

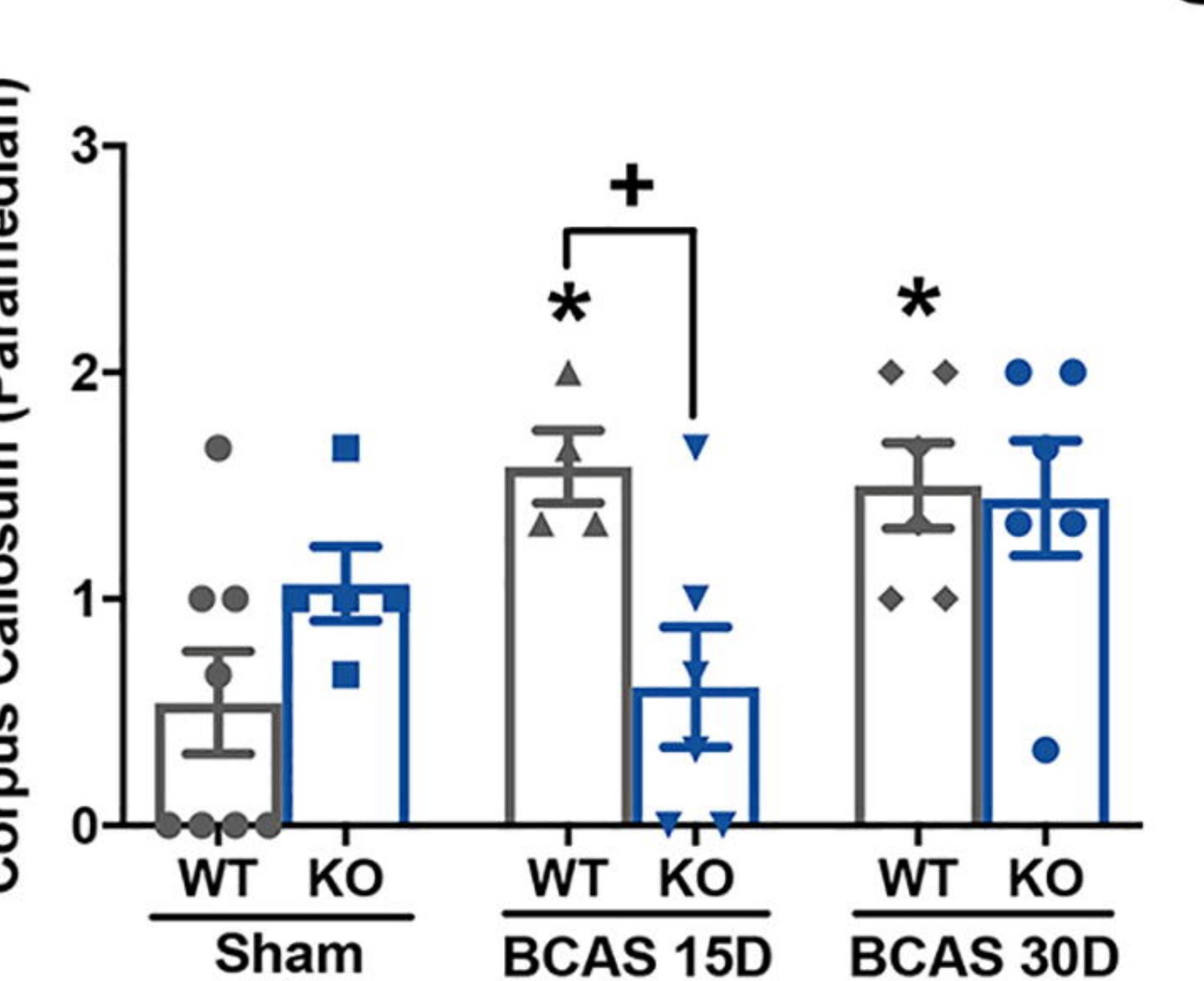
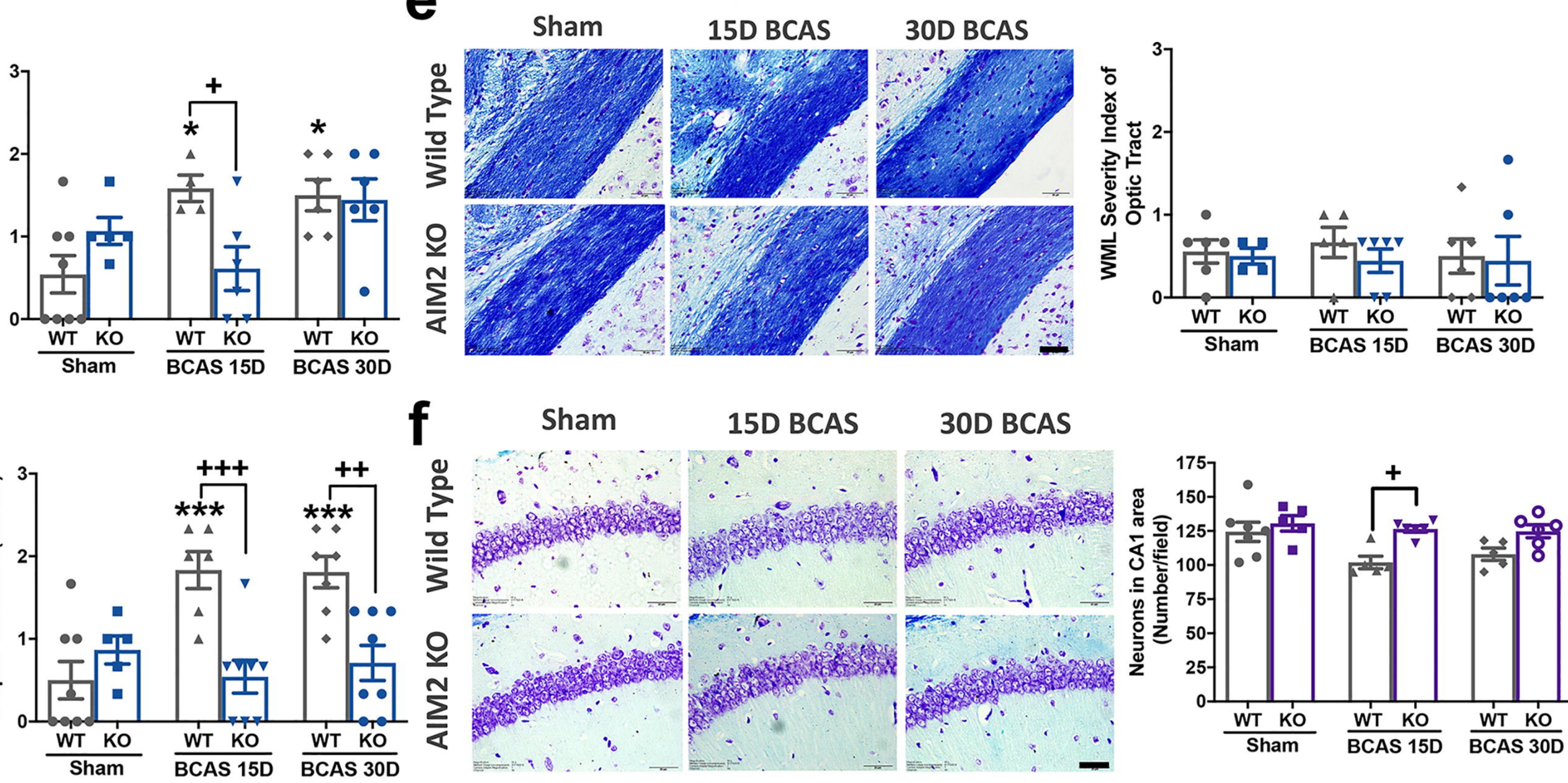

g
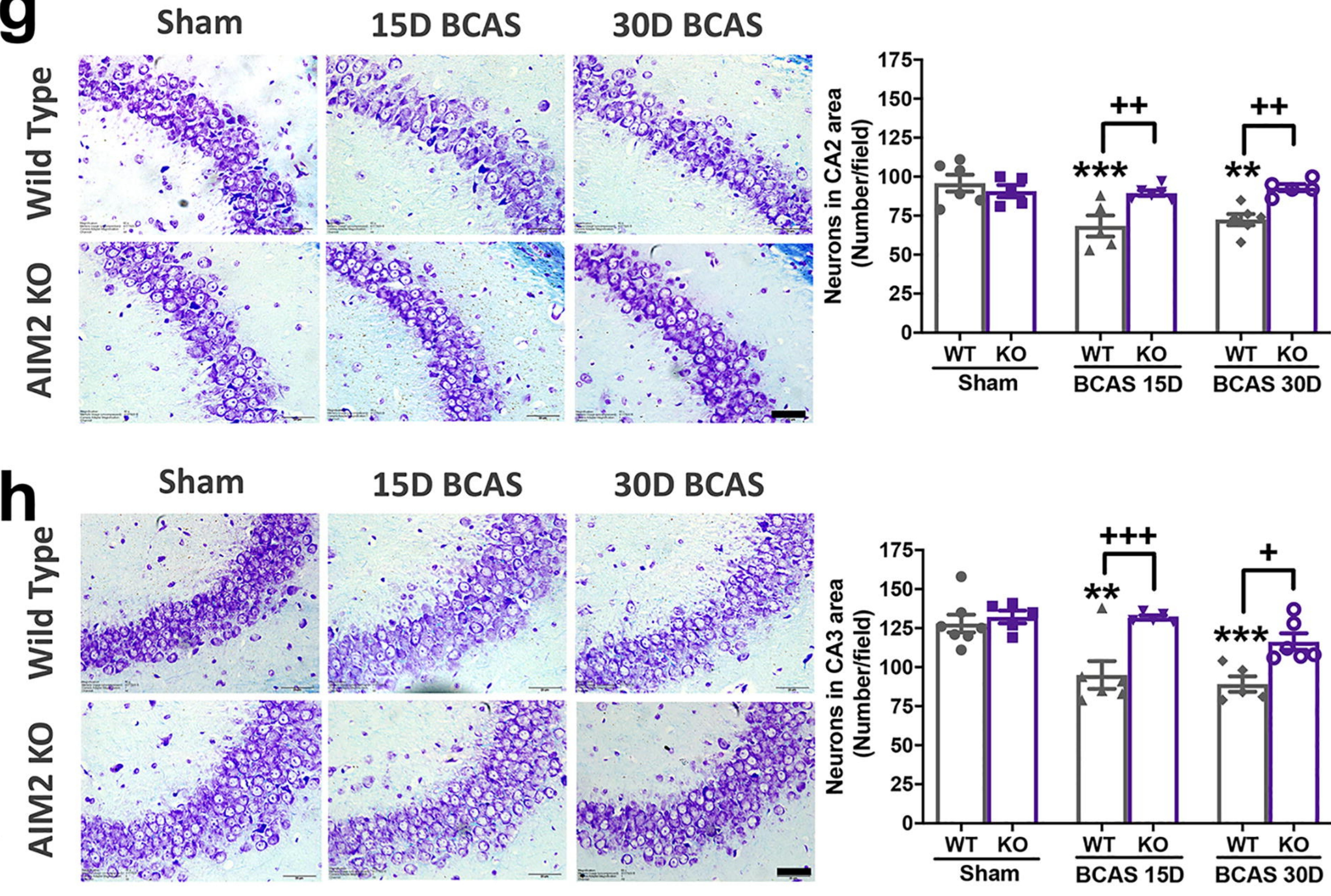
a

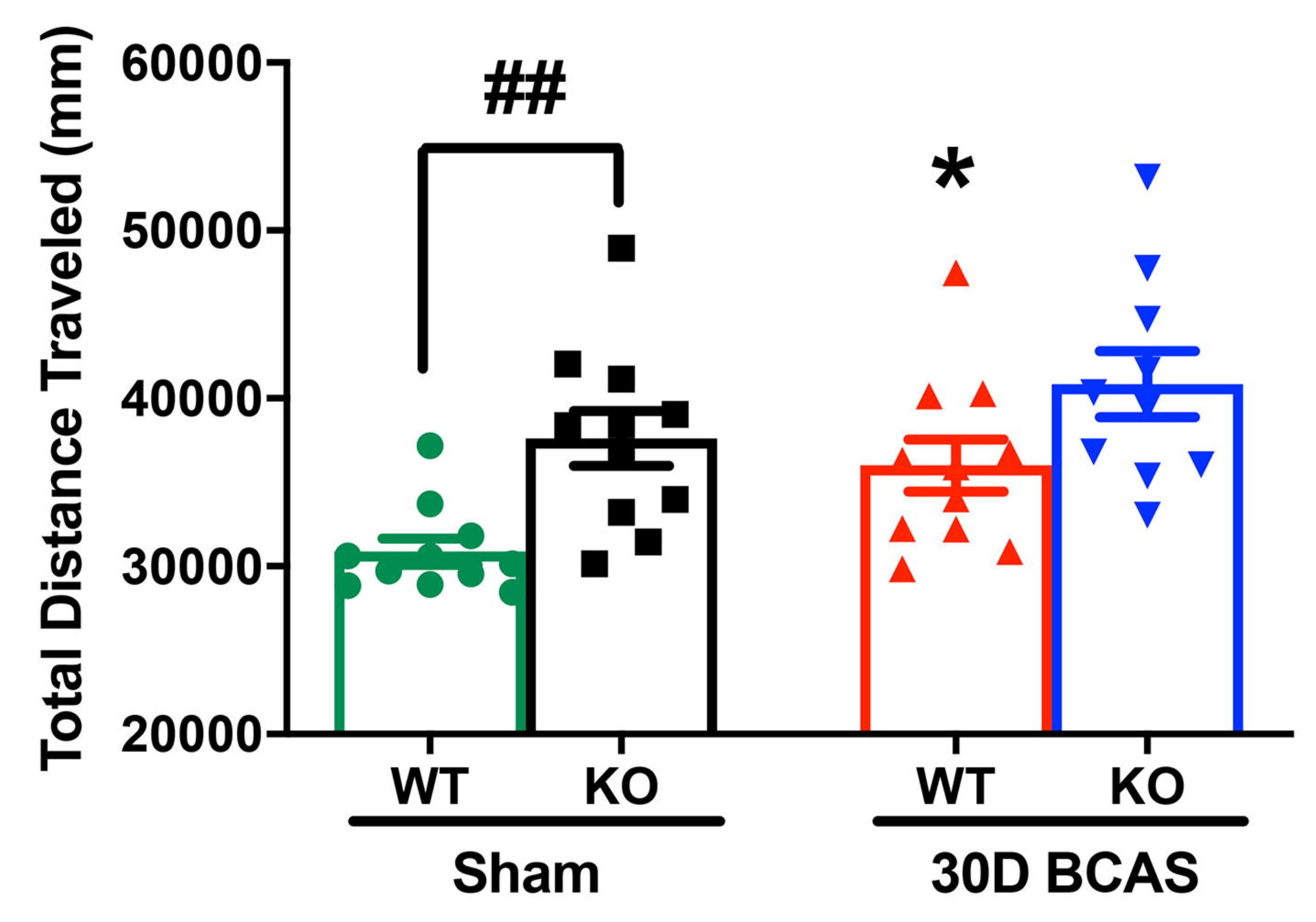

- WT Sham

C
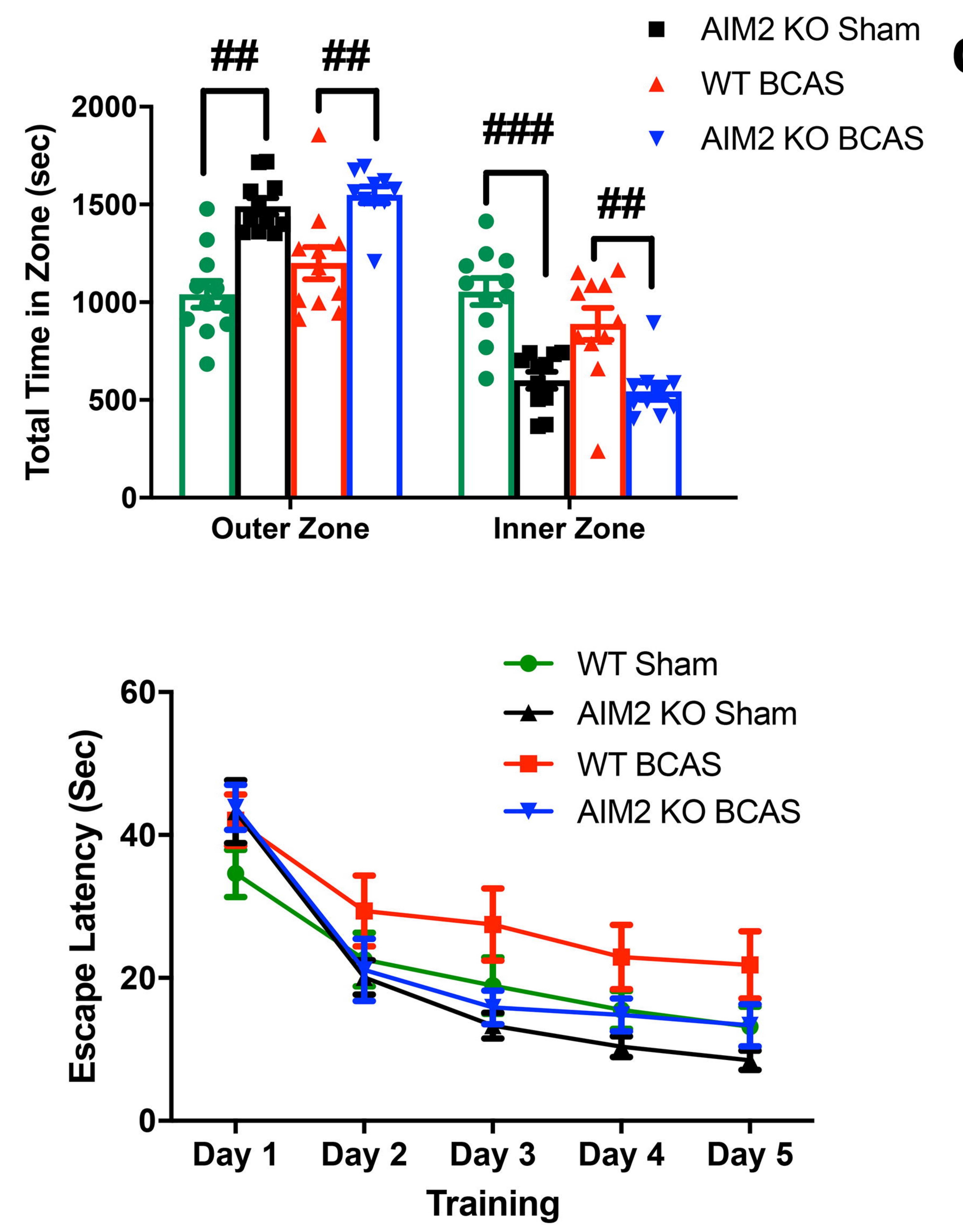

g

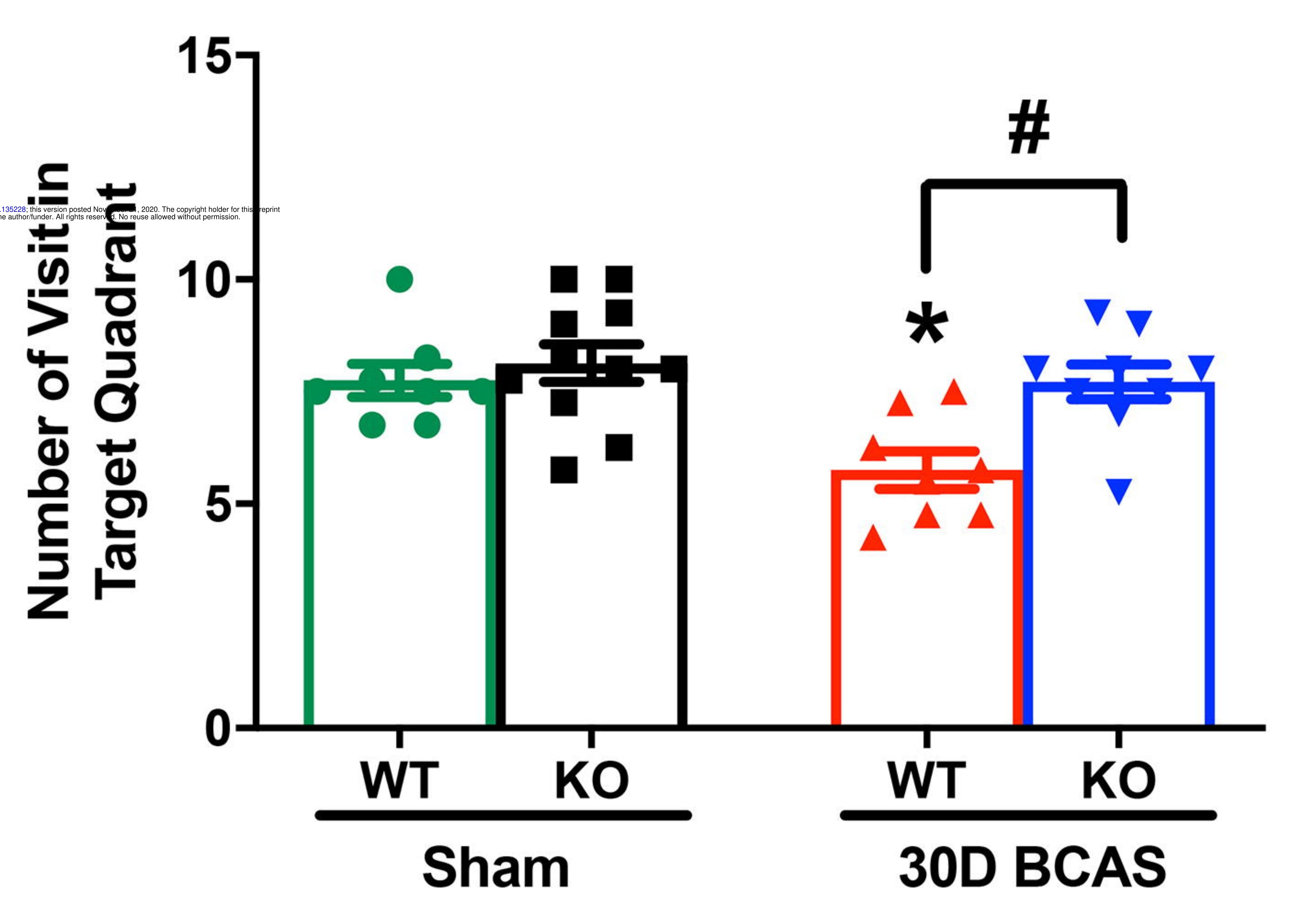

b
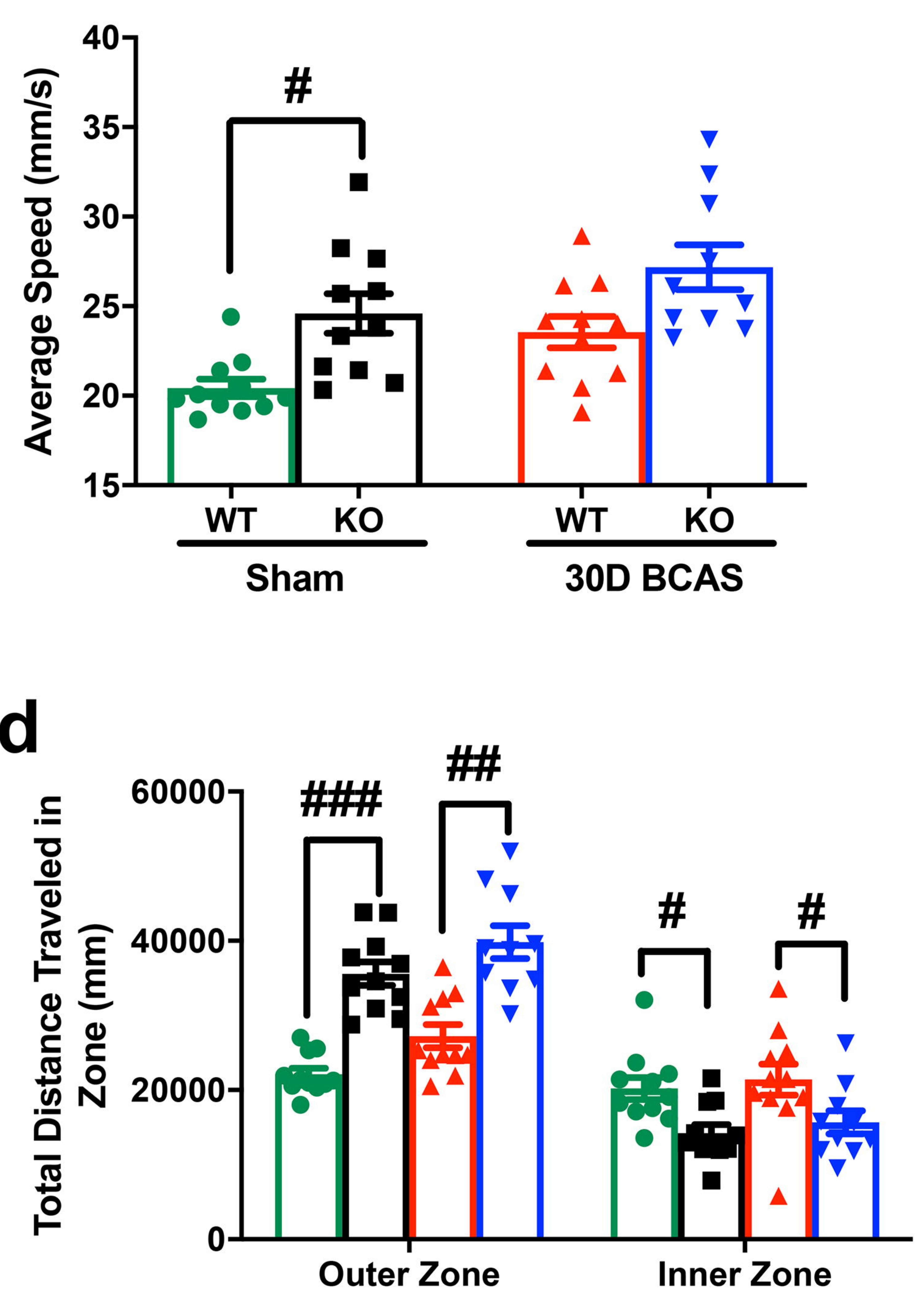

f
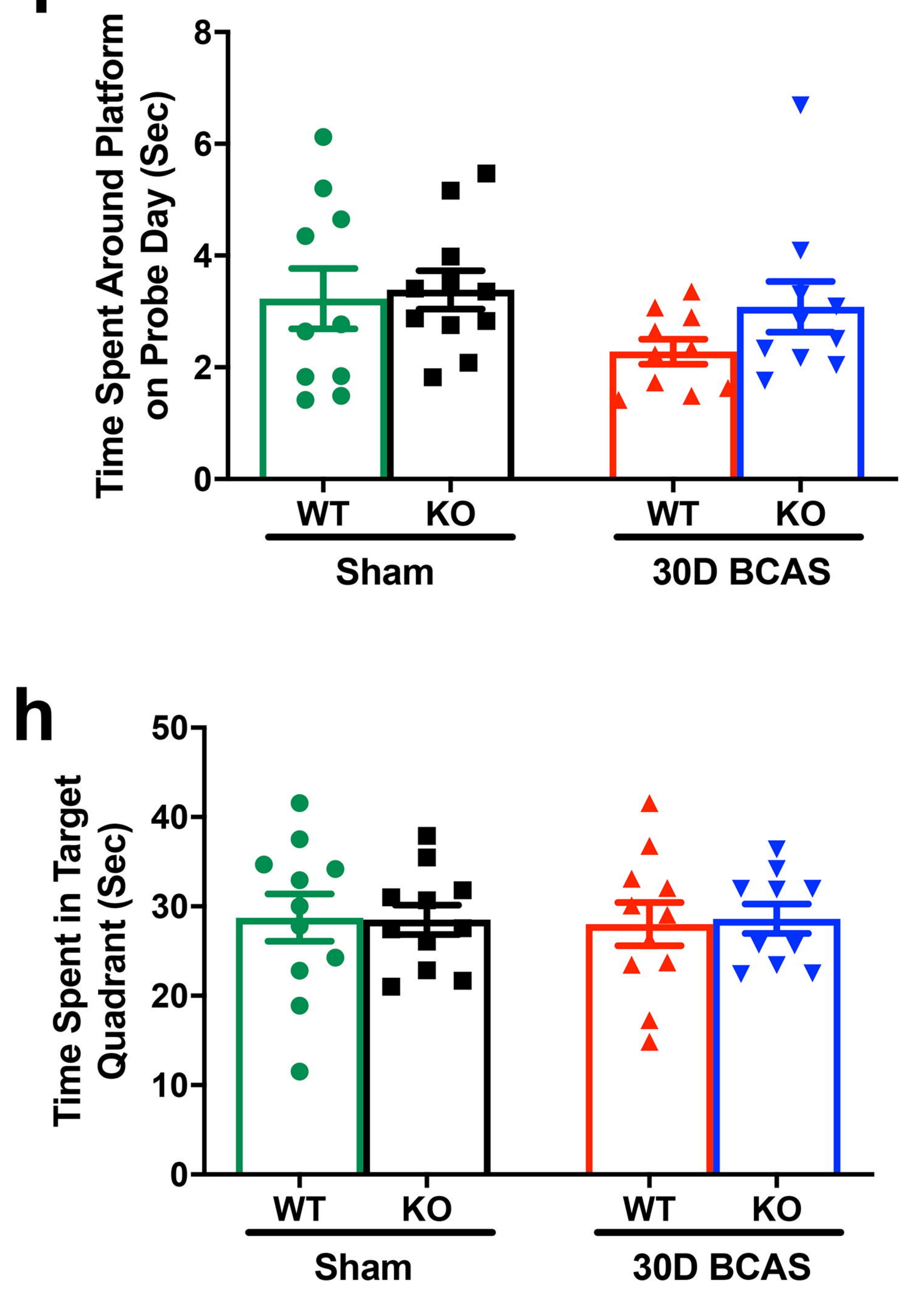